\title{
Early gamma response of sleep is sensory/perceptual in origin
}

\author{
Sirel Karakaș ${ }^{\mathrm{a}, *}$, Orhan Arıkan ${ }^{\mathrm{b}}$, Emine D. Çakmak ${ }^{\mathrm{a}}$, Belma Bekçi ${ }^{\mathrm{a}}$, \\ Elvin Doğutepe ${ }^{\mathrm{a}}$, İlhan Tüfekçi ${ }^{\mathrm{b}}$ \\ ${ }^{a}$ Hacettepe University, Specialty Area of Experimental Psychology, Cognitive Psychophysiology Research Unit, 06532 Beytepe, Ankara, Turkey \\ ${ }^{\mathrm{b}}$ Bilkent University, Department of Electrical and Electronics Engineering, 06800 Bilkent, Ankara, Turkey
}

Received 13 July 2005; received in revised form 17 March 2006; accepted 20 March 2006

Available online 18 May 2006

\begin{abstract}
The goal of the study was to investigate the gamma response of the brain and its functional correlates in rapid eye movements (REM) sleep and the three stages of non-REM sleep. Data on overnight sleep were acquired from 16 healthy, young adult, volunteer males. Neuroelectric activity was recorded from seven recording sites $(\mathrm{Fz}, \mathrm{Cz}, \mathrm{Pz}, \mathrm{F} 3, \mathrm{~F} 4, \mathrm{P} 3, \mathrm{P} 4)$ in response to auditory stimuli $(2000 \mathrm{~Hz}$ deviant and $1000 \mathrm{~Hz}$ standard stimuli: $65 \mathrm{~dB}, 10 \mathrm{~ms} \mathrm{r} / \mathrm{f}$ time, $50 \mathrm{~ms}$ duration) under passive oddball paradigm. Data were analyzed with the Fourier transform and digital filtering and also the recently developed technique of time-frequency component analysis (TFCA). TFCA displayed the gamma response under all stages of sleep. Statistical analysis did not reveal a significant effect of stimulus type, recording site or sleep stage on the three parameters of TFCA, which included maximum value of the time-frequency representation of the extracted gamma component, maximum magnitude of the timedomain representation of the component and the energy of this component. The gamma period included N1 and the early theta response, both of which are related to sensory-perceptual processing in the literature. According to these findings, the gamma response is possibly related, as in wakefulness, to early stimulus processing that also includes sensory/perceptual operations.
\end{abstract}

(C) 2006 Elsevier B.V. All rights reserved.

Keywords: Gamma response; Sleep; Rapid eye movement sleep (REM); Non-REM stages of sleep (NREM); Early stimulus processing; Sensory and perceptual processing; Time-frequency component analysis (TFCA)

\section{Introduction}

The study of brain neuroelectricity using oscillatory dynamics has proven useful in understanding the way brain processes information. One of these oscillations occurs in the gamma band and it is also known as the $40 \mathrm{~Hz}$ rhythm. The present study used the gamma response as a tool for investigating the responses of the brain to exogenous stimuli during rapid eye movements (REM) sleep and stages of NREM sleep.

\subsection{Early gamma response in wakefulness}

Existing literature on the gamma response in wakefulness has reached a level of understanding with respect to the types of

\footnotetext{
* Corresponding author. Tel.: +90 312 2978335; fax: +90 3122992100.

E-mail address: skarakas@hacettepe.edu.tr (S. Karakaș).
}

this oscillation and the function of each type (Gurtubay et al., 2004; Herrmann and Mecklinger, 2001; Herrmann et al., 1999; Lachaux et al., 2000). Reviewing the existing studies, Karakaş et al. (2001) concluded that there are two types of gamma responses in the wakefulness stage and these are discriminated on the basis of temporal localization and time-locking. Of these, the 'early' gamma response occurs within the $150 \mathrm{~ms}$ poststimulus recording time and it is time-locked to the stimulus. The 'late' gamma occurs in approximately 130 $400 \mathrm{~ms}$ time window and it has an induced character.

According to a majority of studies, the early gamma response fulfills a 'sensory' function in wakefulness. This conclusion was reached through studies where gamma response was found in evoked potential recordings over the sensory areas; where it was found to be related to midlatency auditory evoked potentials; and in studies where it was obtained in simple organisms such as the snail and insects (for review, see Galambos, 1992; Karakaş et al., 2001; Başar-Eroğlu et al., 1999). In a recent 
study, Freeman (in press) showed that conditioned stimulus in visual, auditory and somatic modalities evoked early phase transitions over the primary sensory areas in the gamma band. The sensory nature of the early gamma response was also substantiated by Karakaş and Başar (1998) through the utilization of a series of experimental paradigms (evoked potential, single stimulus, easy oddball, hard oddball and mismatch negativity/passive oddball). The comparative analysis of gamma response amplitudes under the different paradigms showed that the early gamma response between $28 \mathrm{~Hz}$ and $46 \mathrm{~Hz}$ did not vary as a function of task complexity, cognitive load or attention allocation. It was concluded from these findings that the time-locked gamma response, which occurred at the early time window of $0-150 \mathrm{~ms}$, was, using the convention of the related literature, a 'sensory' phenomenon.

The brain is basically a parallel processing system that integrates structures/processes that are diffuse but selectively distributed throughout its volume (Başar, 2004; Bressler and Tognoli, in press; Fuster, in press, 1995). The primary, secondary and tertiary association areas, the prefrontal cortex and the limbic system are thus interrelated; accordingly, sensory and perceptual processing are also interrelated. Perception, also called object perception or object recognition, is an operation whereby sensory aspects of the stimulus is associated and matched with existing knowledge and a decision is reached with regards to its identity. Perception thus involves bottom-up processing of stimulus characteristics and top-down processing whereby existing knowledge is brought to bear on the sensory characteristics. This interactional relation results in coherent wholes, gestalts or patterns (Eysenck, 2001; Freeman, in press; Karakaş and Başar, in press).

As would be expected from the close relation between sensory and perceptual processing, gamma response was also obtained when tasks required the recognition of a pattern or object, in other words, under perceptual processing. These studies demonstrated that the gamma response was related to perception of coherent visual patterns, to perception of meaningful visual elements, and served as a neural template (for reviews, see Bașar-Eroğlu et al., 1999; Karakaș et al., 2001). In a recent study, De Pascalis and Cacace (2005) found that phase-ordered gamma oscillation, which was elicited upon painful stimuli, represented pain perception. According to the cinematographic hypothesis (Freeman, in press), stimuli elicited repetitive amplitude-modulation/phase-modulation field patterns; these occurred in discrete frames at the theta band and had the carrier frequencies at the beta and gamma bands. Perception, the formation of gestalts or perceptual wholes, occurs due to scale-free networks of the neocortex, as a result of which it can form almost instantly spatial patterns of any size from several hypercolumns to a whole cerebral hemisphere.

The continuity between sensory and perceptual processes was also suggested by the feature linking or binding hypothesis (Gray and Singer, 1989; Joliot et al., 1994). According to this hypothesis, the mechanism by which coherent patterns are formed operates through the gamma activity. Binding occurs through the spread of the gamma activity in the nonspecific system in a loop between the specific thalamic nucleus and cortical layer 4, feedback from this layer to specific and nonspecific interlaminar nuclei, and feed forward to cortical layers 1 and 5 . The coherency of the gamma waves between these simultaneously active cortical areas allows for the spread of information within the cortex and for the binding of distant brain regions. Sakowitz et al. (2001) found that bisensory stimulation increased the gamma response over multiple sensory areas; these findings show the role of the gamma activity in intersensory association. Bressler (1990) indicated that the gamma wave is a cortical information carrier. The binding hypothesis was supported by findings where gamma decrease was observed in schizophrenia patients in response to nontargets (Haig et al., 2000). Epileptiform spikes were found to desynchronize and diminish gamma activity; spikes were explained as the operation of an "anti-binding" mechanism, which occurs when gamma activity is abnormally intensified (Medvedev, 2002).

The sensory and perceptual nature of the wakefulness gamma response was also demonstrated in studies where the early, time-locked, sensory gamma response co-existed with the perception-related gamma response. Tallon-Baudry et al. (1996) did obtain the phase-locked $40-\mathrm{Hz}$ activity at the early timewindow of $0-200 \mathrm{~ms}$ that did not vary with the stimulus type (the coherent Kanizse triangle, its variant, nontriangle/noncoherent stimulus). Bașar-Eroğlu et al. (1996) obtained the highest gamma during perceptual switching in the active observation of the phenomenon; but it was also obtained under naive observation of the pattern and even in the control EEG. Similarly, in their study on the nonphase-locked late gamma response, Jokeit and Makeig (1994) did obtain the time-locked early gamma response that did not vary as a function of task. These findings show that the task that is employed determines the type of cognitive processing and the type of gamma activation. Depending on the task, the configuration consists of a basic sensory processing and time-locked early gamma response or it may also include perception and induced gamma response. It may thus be concluded that the gamma response of the brain represents sensory/perceptual processing.

\subsection{Gamma activity in sleep}

In the face of these findings on the early, time-locked gamma response in wakefulness, the study of the event-related early gamma response in sleep would have been an appropriate tool for studying the earlier (sensory/perceptual) operations in REM and the stages of NREM sleep. However, save that by Llinas and Ribary (1993), gamma response has been a neglected area in sleep research. Evoked potential recordings in this study indicated that the thalamocortical system was accessible to sensory input in both waking and REM. Gamma activity was observed in the spontaneous activity of both stages. However, gamma activity was not reset by sensory stimuli in REM (Llinas and Ribary, 1993); it did not become enhanced upon stimulation. This finding was evaluated as 'the turning away of attention' from sensory input in REM toward the functional state being generated by the brain at that time. In 
REM, sensory input could not activate conscious experience and external world was thus not perceived (Llinas and Pare, 1991).

Meanwhile, abundant research on the event-related potentials (ERPs) in REM and the stages of NREM sleep demonstrated that there is responsiveness to external stimuli which may, however, vary according to stages of sleep (MullerGass and Campbell, 2002). These studies showed that N100 and the mismatch negativity component (MMN), the primary ERP responses that represent sensory-perceptual processing, were obtained in sleep in response to external stimuli. Lack of sensory reset of the gamma response contradicts also the other findings that show responsiveness in REM. One is the wellknown phenomenon in which stimulation is incorporated in REM into the dream content (for a review, see Burton et al., 1988). It was demonstrated that meaningful stimuli, which also require less intensity to evoke an arousal from any stage of sleep (Oswald et al., 1960), produce P300 responses during REM and Stage 2 sleep (for a review, see Cote and Campbell, 1999). Such findings indicate that lack of reset in REM should at least not be absolute but related to the characteristics, significance and/or salience of stimuli (Brualla et al., 1998; Perrin et al., 1999). The elicitation of the parietal P300 response to at least the rare, intrusive or meaningful stimuli showed that REM, a period of endogenous stimulus processing related to dreaming, may involve extended processing of exogenous stimuli under specific task conditions.

\subsection{Aim of the study}

The extent to which the sleeping brain can sense and integrate external and internal stimuli has been among the most intriguing subjects of sleep research. Literature shows that, in wakefulness, the gamma response is related to sensory/ perceptual processing. The existence and the function of the gamma response in sleep is largely a neglected and still unresolved issue of the literature. The present study used the gamma response as a tool for understanding the responsiveness of the brain to external stimuli during sleep and tested the hypothesis that this response represents, as in wakefulness, sensory/perceptual processes.

\section{Methods and materials}

\subsection{Participants}

Data on overnight sleep was collected from $16(\overline{\mathrm{x}}=24.69 \pm$ 4.42 , range: $18-33$ years) right-handed, naive males. They had at least 11 years of education (senior high school education). Participants gave informed consent to participate in the study after the purpose and nature of the experiment was fully explained. Participants were advised to abstain from alcohol and caffeine on the evening of the study. Of the sample, 56.3\% consumes alcohol. In this subsample, frequency of the incidence of alcohol consumption varied between 1 and 8 times per month $(44.4 \%$ and $11.1 \%$, respectively; $2.67 \pm$ 2.34). The last incidence of alcohol consumption varied between 3 and 60 days $(11.1 \%$ and $11.1 \%$, respectively; $17.22 \pm 20.04)$. The last occasion of coffee and/or tea consumption varied between $1 \mathrm{~h}$ and $24 \mathrm{~h}(14.3 \%$ and $7.1 \%$; $5.75 \pm 5.67)$. The proportion of the sample who smokes was $50 \%$. Frequency of usage was between 5 and 20 cigarettes per day $(12.5 \%$ and $37.5 \%$, respectively; $14.37 \pm 5.47)$. The last incidence of smoking varied between $30 \mathrm{~min}$ and $12 \mathrm{~h}(25 \%$ and $12.5 \%$, respectively; $3.00 \pm 3.84$ ).

The sample included individuals who reported themselves as good sleepers with regular sleep-wake patterns. Individuals with neurological and/or psychiatric disorders, those who were taking or who recently stopped taking antidepressants or psychotherapeutic drugs were not admitted to the study. Individuals who were currently using drugs that could affect cognitive processes or who recently stopped taking such drugs were also not admitted to the study. Hearing level of potential participants was assessed through computerized audiometric testing prior to the experimental procedures. Individuals with hearing deficits were not employed in the study.

\subsection{Stimuli, paradigms and procedures}

The acoustical sine waves which had $10 \mathrm{~ms} \mathrm{r} / \mathrm{f}$ time and $50 \mathrm{~ms}$ duration were presented over two loudspeakers that were placed at a distance of $2.40 \mathrm{~m}$ from the participant. The loudspeakers were $1.75 \mathrm{~m}$ apart and were so located as to have participant's head at approximately the center. The stimuli were presented at an intensity of $65 \mathrm{~dB}$ SPL near the participant's head. There were two types of stimuli: the standards and the deviants. Standard stimuli were $1000 \mathrm{~Hz}$ and the deviant stimuli were $2000 \mathrm{~Hz}$. The deviants $(p=.22)$ were embedded randomly within a series of frequent standards $(p=.78)$. The stimuli were organized into blocks of 200 standard and 55 deviant stimuli. These blocks were repeated so long as the participant slept. The specified stimulus conditions define the standard oddball (OB) paradigm of cognitive psychophysiology. Since, due to the nature of the state of consciousness, the participant did not perform an intentional discriminatory response, the paradigm was called passive OB (OB-p).

For sleep studies, participants arrived at the laboratory approximately $2 \mathrm{~h}$ prior to their reported sleep time. In this interval of time, participants were oriented to the conditions of the study. Natural sleep duration of participants were reported to vary between 5 and $8 \mathrm{~h}(6.3 \%$ and $12.5 \%$, respectively; $6.42 \pm .90$ ). The sleep duration in the experimental study was found to vary between 4.5 and $8 \mathrm{~h}(6.7 \%$ and $13.3 \%$, respectively; $6.77 \pm 1.00$ ). The beginning of the sleep episodes occurred between 23:00 and 1:00; the end of the sleep episodes occurred between 6:30 and 8:55. Fifty percent of the sample woke up spontaneously, the rest had to be woken up. Eighty one percent of the sample reported to have slept comfortably.

Data of the present study represent first night recordings. Participants spent a single night in the laboratory, lying on a bed that was situated in a dimly illuminated and ventilated sleep 
chamber. The chamber was electrically and acoustically shielded. Participants were allowed to fall asleep and stimulation started after consolidated sleep onset, defined as approximately 5 min of uninterrupted Stage 2 sleep, and continued throughout the night.

\subsection{Electrophysiological recording}

Brain's neuroelectricity was recorded at 7 recording sites (Fz, Cz, Pz, F3, F4, P3, P4) of the 10-20 system using Ag/ $\mathrm{AgCl}$ electrodes. Electrodes were referenced to linked earlobes with forehead as the ground. Additional electrodes were placed at the outer canthus and supraorbitally at the left eye for bipolar recording of electro-ocular (EOG) activity. Bipolar recording was also made of submental electromyographic (EMG) activity. The impedance for all electrode sites was 3 $\mathrm{k} \Omega$ or less. The EEG was amplified and filtered with a bandpass between 0.16 and $70 \mathrm{~Hz}$ ( $3 \mathrm{~dB}$ down, $12 \mathrm{~dB} /$ octave) (Nihon Kohden Neurofax 4418K). Notch filter was not activated.

Total recording time was $2000 \mathrm{~ms} ; 1000 \mathrm{~ms}$ of this period served as the prestimulus baseline. The period between two consecutive recording periods (epochs) randomly varied between $500 \mathrm{~ms}$ and $1500 \mathrm{~ms}(\overline{\mathrm{x}}=1000 \mathrm{~ms})$. The pre- and poststimulus activity was digitized with a sampling rate of $500 \mathrm{~Hz}$.

EEG/ERP data acquisition, analysis, and storage were achieved by a commercial system (Brain Data 2.80), which also controlled stimulus presentation and automatic rejection of trials in which response amplitudes exceeded $\geq 100 \mu \mathrm{V}$. This system was also used for the on-line monitoring of the single sweeps and the averaged ERP waveforms. EEG, EOG and EMG were monitored throughout the night; recordings were also made of these activities for off-line EEG evaluation. The gross behavioral responses of participants were monitored through CRT of the Neurofax (Nihon Kohden EEG Formatter VY-210BK). Responses that could alter stimulation intensity (e.g., covering the ears) or have negative effect on the records (e.g., movements, snoring) were noted on the EEG recording sheet. Sweeps that fell into the duration of such states were not included in the analyses.

Sweeps were staged in the present study by concurrent usage of EEG, EOG and EMG. Analyses were performed on epochs that were embedded in homogeneous stages of sleep. For Stage 2 sleep, epochs embedded in spindles $(12-14 \mathrm{~Hz})$ were chosen. Epochs with K-complexes were not included in the analyses. Accordingly, the label Stage 2 refers in the present study to a subset of phenomena that characterize this stage. For Stage 3 sleep, the requirement was $20-50 \%$ EEG delta activity $(\leq 2 \mathrm{~Hz})$. The requirement for Stage 4 sleep was a high proportion of delta activity (70-100\%). For REM, high-frequency low-voltage EEG activity, EOG activity and reduced EMG was required. Sweeps where sleep stage was ambiguous, where there were stage changes or extensive muscle artifacts, were excluded from any further analysis. The scoring was found to have an interrater agreement around $95 \%$.

\subsection{Data analyses}

\subsubsection{Time-domain analysis}

Before the averaging procedure, the sweeps that contained artifacts were rejected first by on-line (automatic rejection of sweeps with amplitudes $\geq 100 \mu \mathrm{V}$ ) and later off-line technique. Epochs with high-amplitude sporadic muscular activity and eye movements, those that did not meet stagespecific criteria, were coincident with stage changes or ambiguous stages were rejected through visual inspection of the continuous EEG-EOG-EMG recordings. Selective averaging was applied to ERP data to obtain representative waveforms and to separate the ERP (evoked activity) from the superimposing EEG (spontaneous activity). Average ERPs for single participants were calculated from their artifact-free sweeps. Grand average ERPs were calculated from all artifactfree sweeps.

\subsubsection{Frequency-domain analysis}

Frequency-domain responses were obtained using two methods: amplitude frequency characteristics and digital filtering (DF). The amplitude frequency characteristics (AFC) were computed using the method of transient response frequency characteristics (TRFC) (for details of methodology, see Karakaş and Başar, 1998). In TRFC, the amplitude frequency characteristics, $|G(j \omega)|$, is computed by the application of the Laplace transform (i.e., one-sided Fourier transform) to the unfiltered transient (evoked) response, $c(t)$, of the system. The AFC is expressed in relative units and it reflects the amplification in the studied frequency channels. The presence of peaks in the AFC reveals the frequency responses of the system and these are interpreted as the most preferred oscillations of the system when responding to stimuli. The AFC was thus used in the present study for a global and simultaneous description of responses in the studied frequency range $(1-100 \mathrm{~Hz})$. The AFCs were also used for determining the limits of the frequency responses that appeared as maxima in the AFCs and these were later used for determining the cutoff values of the digital filters.

In DF, the experimentally obtained transient (evoked) response, $c(t)$, was filtered by means of the convolution integral using the weighting function, $g_{\mathrm{KF}}(t)$, of (for details of methodology, see Farwell et al., 1993). Digital filtering produces visual displays of the time courses of oscillatory responses within the frequency limits of the utilized filters over the studied time intervals.

In the present study, digital band-pass filtering was responseadaptive; filter limits were defined as the low- and highfrequency limits of the maxima in the AFCs. Accordingly, the cutoff values of the digital filters coincided with the limits of the maxima in the AFCs. In the present study, the high and low cutoff limits of the filters were separately obtained for each combination of experimental conditions which comprised of four stages of sleep (REM, three stages of NREM sleep), three recording sites $(\mathrm{Fz}, \mathrm{Cz}, \mathrm{Pz})$, and two types of stimulus (deviant, standard). 


\subsubsection{Time-frequency domain analysis}

The simultaneous localization of signals in the time and frequency planes can be achieved using short-time Fourier transform (STFT). However, when ERP components are closely localized in the time-frequency plane, STFT may not adequately resolve them. Wigner distribution significantly improves the resolution of the individual ERP components. The Wigner distribution $W_{x}(t, f)$ of a signal $x(t)$ is defined as

$W_{x}(t, f)=\int_{-\infty}^{\infty} x(t+\tau / 2) x^{*}(t-\tau / 2) e^{-j 2 \pi f \tau} \mathrm{d} \tau$.

As it is seen from the definition, Wigner distribution is a bilinear representation. Therefore, Wigner distribution of multicomponent signals or mono-component signals with curved time-frequency supports will be cluttered by spurious terms called cross-terms. The existence of cross-terms may decrease the interpretability of the time-frequency distribution. ERPs, like other non-stationary signals, require an analysis technique that is free from cross-terms and that also renders a highresolution time-frequency distribution.

The simultaneous localization of the gamma component in the time and frequency domains was achieved in the present study using the recently developed time-frequency component analysis (TFCA) technique (Özdemir et al., 2005). TFCA extracts the components of a multi-component signal in a crossterm free manner and computes high-resolution time-frequency distribution of the composite (multi-component) signal. Using the fractional domain warping concept, TFCA can identify the auto-terms with linear or curved time-frequency supports in the time-frequency plane.

TFCA identifies and extracts the maximal energies of the oscillatory components from the composite (multi-component) ERP signal (Karakaş et al., in press; Özdemir et al., 2005). Accordingly, this high-resolution signal analysis technique can produce the global distribution of signal components in the form of spatially and temporally integrated, time-varying oscillatory activity of various frequency ranges. Unlike Fourier transform and the respective amplitude frequency characteristics, TFCA does not assume that the system is linear or stationary. The requirement in TFCA is the existence of signal components that have non-overlapping supports in the time-frequency plane.

\subsubsection{Detection of early gamma response based on time- frequency domain characteristics}

Detection of the time-locked early gamma response was realized using an automated detection technique (for details, refer to Karakaş et al., in press).

The major steps of the detection algorithm were (1) preprocessing of the EEG data to enhance detection; preprocessing involves filtering out EEG signals outside 28 $46 \mathrm{~Hz}$ band, and time weighting around the temporal support of the early gamma response; (2) computing short-time Fourier transform (STFT) of the pre-processed signal; (3) locating time-frequency domain peaks that would be due to the potential presence of an early gamma response; (4) identifying the frequency interval of each detected peak; (5) filtering the EEG recording within the identified frequency intervals in Step 4; (6) computing two STFTs: (a) STFT of the signal obtained in Step 5: $S_{y_{i}}(t, f)$; (b) STFT of the signal obtained in Step 5 after time weighting: $S_{g_{i}}\left(t_{f} f\right)$; here $i$ denotes the index of the potential early gamma response; (7) extracting the support marker for $S_{g_{i}}(t, f)$ : $I_{g_{i}}\left(t_{f}\right)$; (8) forming the support marker for $S_{y_{i}}(t, f)$ by using $I_{g_{i}}(t, f)$ and pre-stimulus region boundaries; (9) extracting quantitative time-frequency domain features; and (10) detecting the early gamma response based on the extracted features.

To illustrate the efficiency of the extracted features, a neural network (NN) classifier with 3 inputs (extracted features) and a single output (indicating the presence of the early gamma response) was designed. The neural network had two hidden layers, with 30 and 15 neurons respectively. The transfer functions in all of the layers were hyperbolic tangent transfer functions.

The designed neural network was trained (using backpropagation algorithm) on a set that contained 21 signals that exhibited gamma response, $\mathrm{G}^{+}$, and 14 signals that did not exhibit gamma response, $\mathrm{G}^{-}$. Preliminary classification of the signals into $\mathrm{G}+$ and $\mathrm{G}-$ were achieved through expert opinion. The trained network was tested on 102 signals, which were recorded in response to the deviant and standard stimuli from the different recording sites. There was an agreement of $76 \%$ between the classifications of the expert and the preliminary neural network (G+ concordance: $47.06 \%$; $\mathrm{G}^{-}$concordance: $29.41 \%$ ). The false-positives represented $7.84 \%$ and the false negatives, $15.69 \%$ of the total number of cases (Karakaş et al., in press).

\section{Results}

The sweeps that were obtained in response to the deviant stimuli consisted of artifact-free ones. For a comparable signalto-noise ratio, a similar number of sweeps were randomly chosen from all the artifact-free sweeps that were obtained in response to the standard stimuli. The number of sweeps for the deviant stimuli was as follows: Stage 2 (922), Stage 3 (651), Stage 4 (2333), REM (1402). The number of sweeps for the standard stimuli was as follows: Stage 2 (1083), Stage 3 (691), Stage 4 (2500), REM (1593).

\subsection{Amplitude frequency characteristics, event-related potentials and filtered event-related potentials}

Fig. 1 shows the amplitude frequency characteristics (AFC) of the grand average ERPs recorded from midline recording sites (Fz, Cz, Pz) under NREM stages of sleep (Stages 2-4) and REM. The first graph in each pair of graphs shows the AFC for the deviant-elicited ERP and the second one, for the standardelicited ERP.

According to Fig. 1, the AFCs for both the deviant and standard stimuli included gamma range activity in REM. In NREM stages of sleep, gamma activity was not as distinct. The AFCs for the lower frequencies showed three different patterns. One configuration included differentiated delta and distinct 
$\mathrm{Fz}$

Stage 2

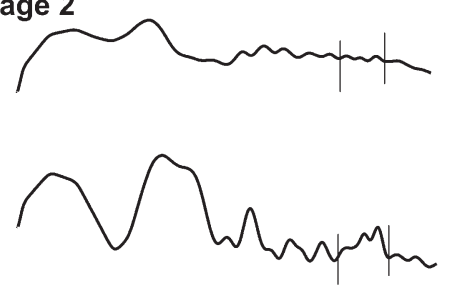

Stage 3

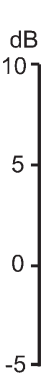

Stage 4

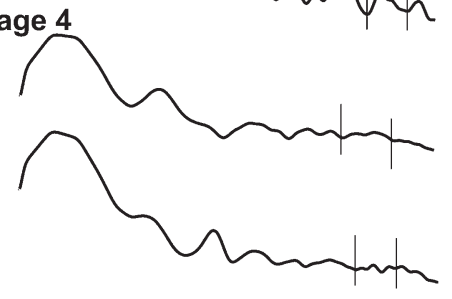

$\mathrm{Cz}$
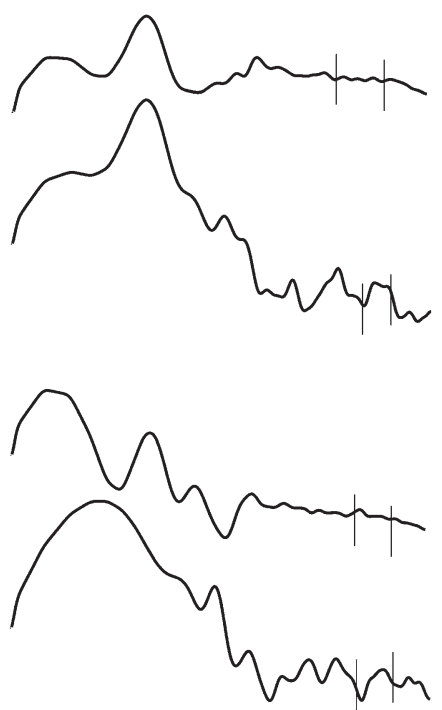
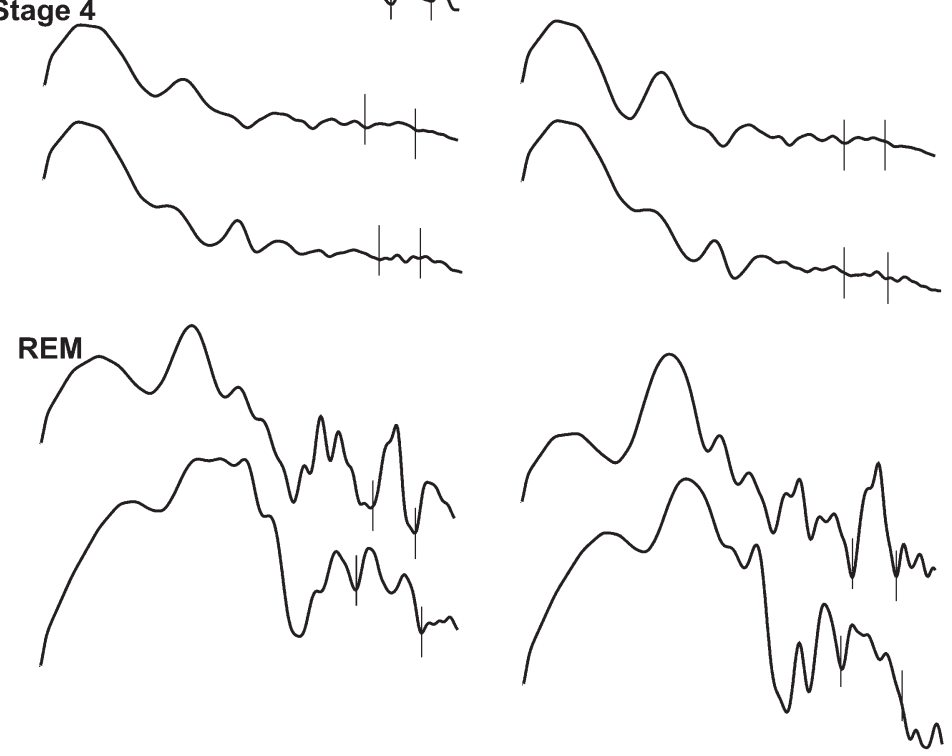

$\mathrm{Pz}$
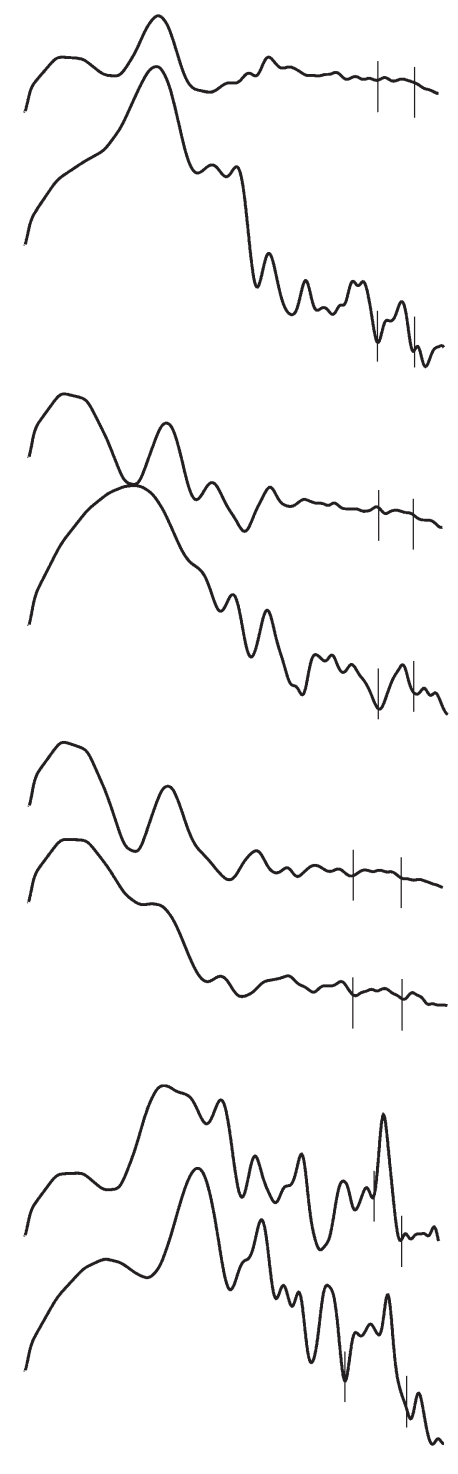

$\begin{array}{lllllllllll}1 & 2 & 3 & 5 & 7 & 10 & 20 & 30 & 5070 \mathrm{~Hz}\end{array}$

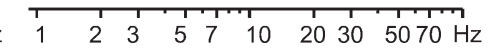

$\begin{array}{lllllllllll}1 & 2 & 3 & 5 & 7 & 10 & 20 & 30 & 5070 \mathrm{~Hz}\end{array}$

Fig. 1. Amplitude frequency characteristics (AFC) of the grand average ERPs ( $n=16)$ calculated for $\mathrm{Fz}, \mathrm{Cz}$ and Pz recording sites (columns $1-3$, respectively) for NREM Stages 2-4 and REM. In each block, the first AFC is for the deviant and the second one for the standard stimuli. Curves were obtained by applying Fourier transform to averaged ERP curves that were obtained in the $0-1000 \mathrm{~ms}$ poststimulus recording time. Vertical lines in the AFCs show the frequency limits of the gamma range activity; these defined the cutoff frequencies in digital filtering. Abscissa: frequency in logarithmic scale. Ordinate: potential amplitude (gain), $|G(j \omega)|$, in decibels. The curves were normalized in such a way that the amplitude at $0 \mathrm{~Hz}$ was equal to 1 (or $20 \log 1=0$ ).

theta responses and these were obtained in response to deviant stimuli under Stages 2-4. A second configuration included a broad frequency band that peaked at the theta range; this was obtained in response to deviant stimuli under REM and to standard stimuli under REM and Stage 2. The third configuration was a broad frequency band that peaked at the delta range; this was obtained in response to the standard stimuli under Stages 3 and 4.

The first waveform in each block of figures in Figs. 2 and 3 present the ERPs obtained from the $\mathrm{Fz}, \mathrm{Cz}$ and $\mathrm{Pz}$ recording sites in response to the deviant stimuli and standard stimuli, respectively, under NREM Stages 2-4 and REM.
Overall, the ERPs in sleep stages showed two negative peaks, two positive peaks and a late negative peak. The peaks were labeled in the present study using polarity and order of occurrence as N1, P2, N2, P3, N3 (Figs. 2 and 3). The only ERP peak that occurred in the time window of the early gamma response in REM and all stages of NREM sleep was N1. The average peak latency of N1 varied between $94 \mathrm{~ms}$ and $148 \mathrm{~ms}$ (134.13 \pm 22.88$)$.

The effect of sleep stage (REM and NREM Stages 2, 3 and 4), recording site (Fz, Cz, Pz, F3, F4, P3, P4) and stimulus type (deviant, standard) was tested on the amplitude of the N1 peak using a $4 \times 7 \times 2$ analysis of variance (ANOVA) with repeated 


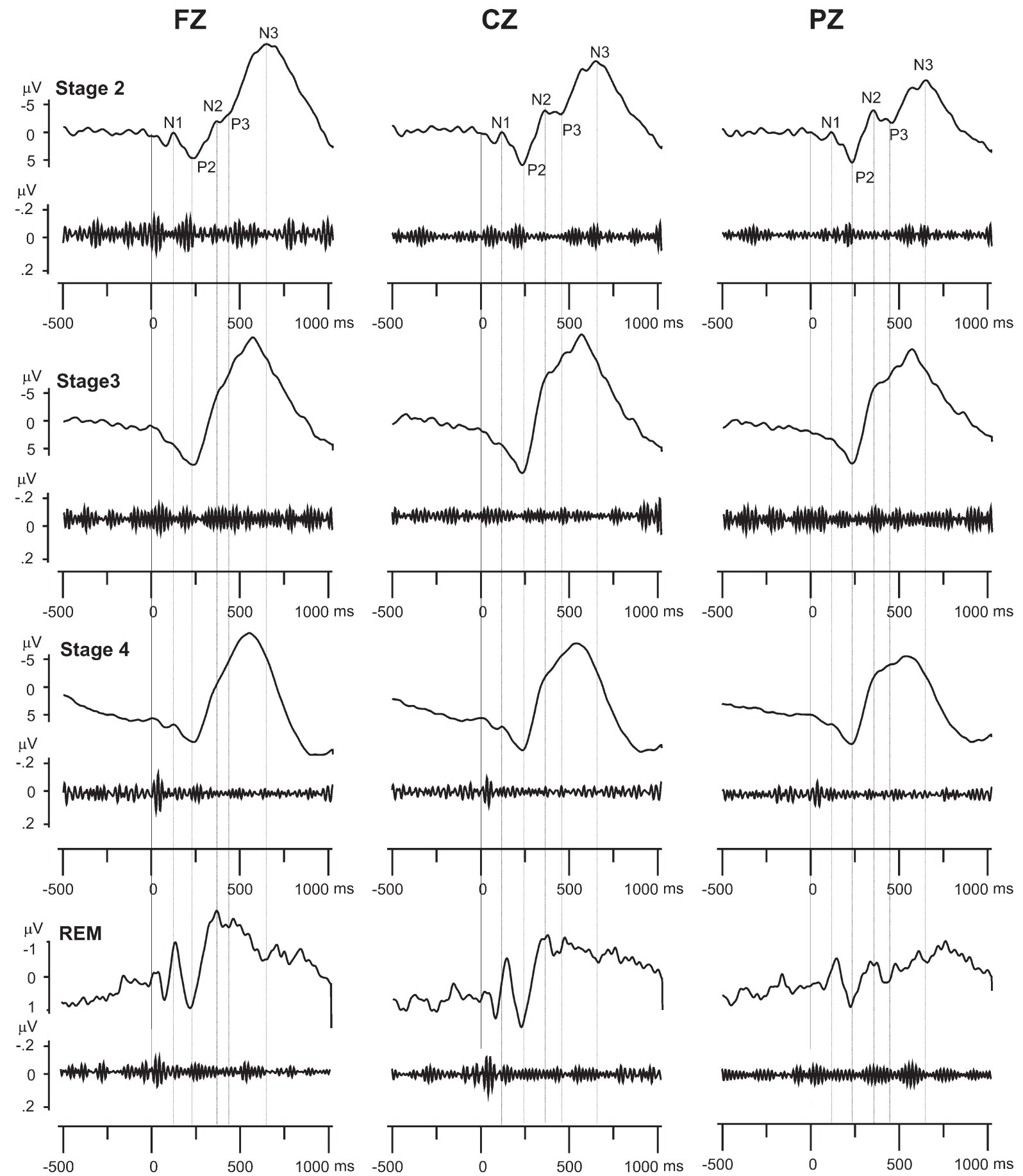

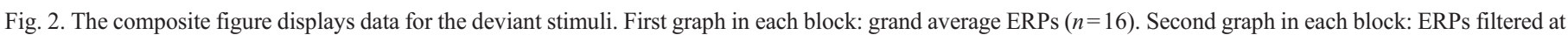

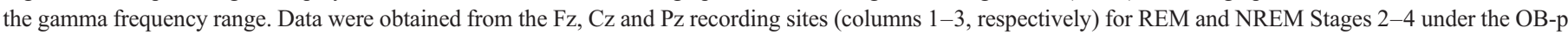
paradigm. Stimulation applied at ' 0 ms' time point. Vertical lines denote stimulus onset and the latencies of the ERP peaks as referenced to Stage 2.

measures on all factors (Vasey and Thayer, 1987). As is required by ANOVA, the dependent variable (voltage values) comprised of the ratio scale. Some of the data did not satisfy the assumption of normality. Different transformation techniques could not achieve distributions that satisfied the normality assumption. Under this circumstance, univariate outlier rejection was performed and the data were standardized using $z$ transformation. Equality of variances was checked by test of sphericity; Mauchly's test showed that the data were spherical.
Using the values for spherical data, the effect of recording site was found significant, $F(6,30)=3.112, p=.017$. The remaining main and interaction effects were found to be nonsignificant. Post hoc pair-wise comparisons were made using Bonferroni method for control of Type I error. These analyses did not find significant differences in N1 amplitudes that were obtained from the different recording sites.

The second waveform in each block of figures in Figs. 2 and 3 present the findings on the ERPs that were filtered (f- 
FZ
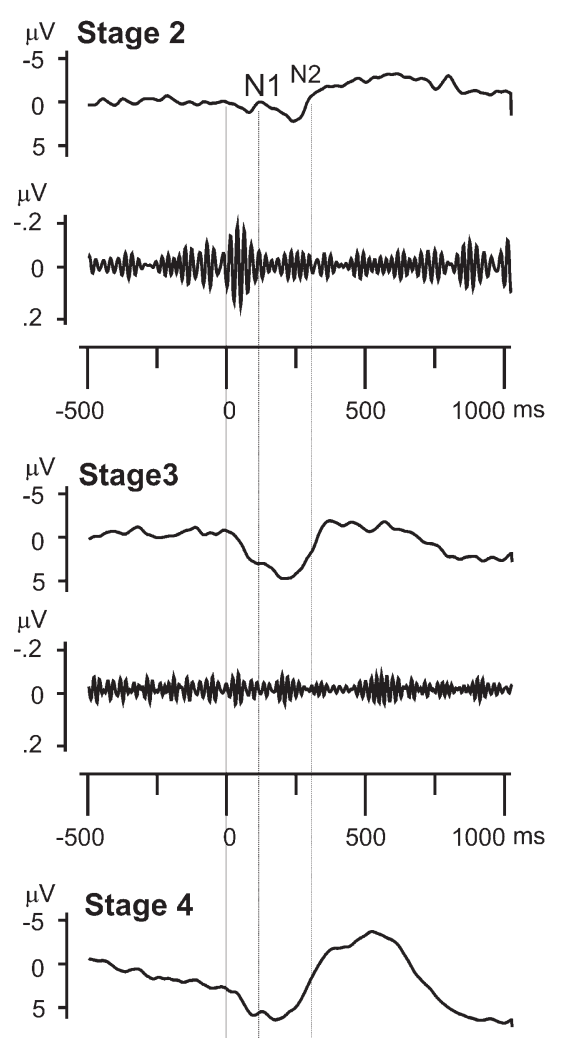

$\mu \mathrm{V}$
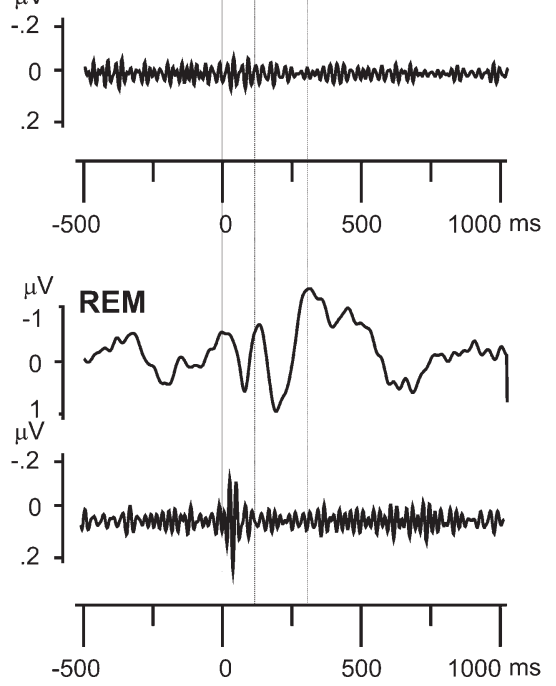

CZ
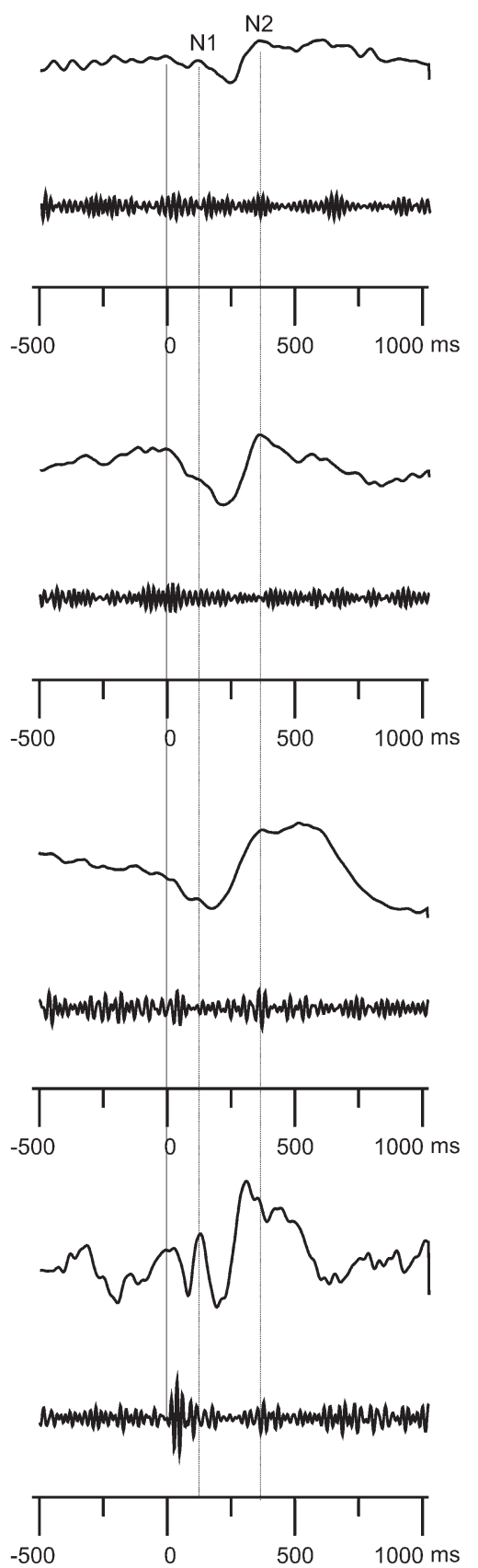

PZ
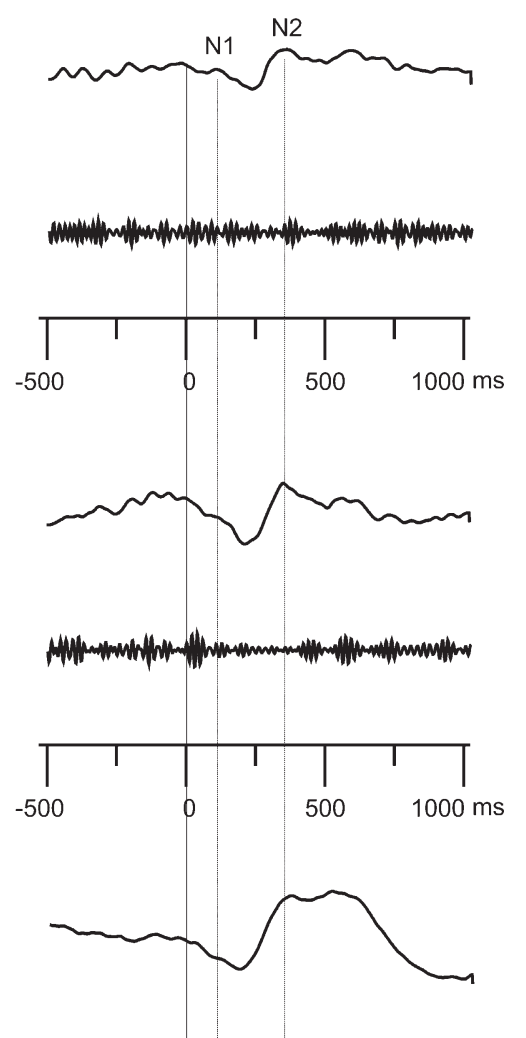

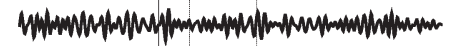
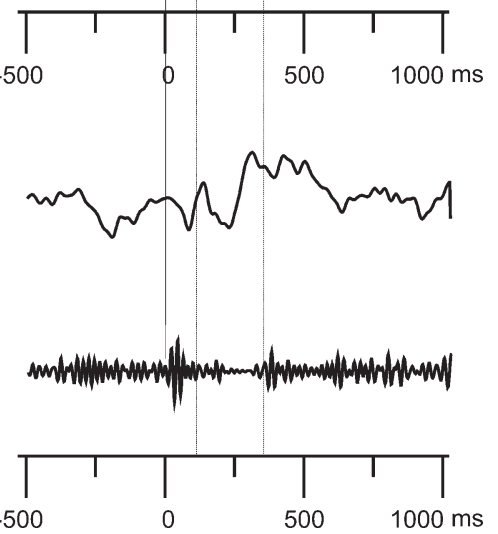

Fig. 3. The composite figure displays data for the standard stimuli. First graph in each block: grand average ERPs $(n=16)$. Second graph at each block: ERPs filtered at the gamma frequency range. Data were obtained from the Fz, Cz and Pz recording sites (columns 1-3, respectively) for REM and NREM Stages 2-4 under the OB-p paradigm. Stimulation applied at ' $0 \mathrm{~ms}$ ' time point. Vertical lines denote stimulus onset and the latencies of the ERP peaks as referenced to Stage 2.

ERPs) in the gamma frequency range. Fig. 2 includes the fERPs that were obtained for the deviant stimuli and Fig. 3 includes the f-ERPs that were obtained for the standard stimuli for the $\mathrm{Fz}, \mathrm{Cz}$ and $\mathrm{Pz}$ recording sites under NREM Stages $2-4$ and REM. Filter limits were separately obtained for each combination of experimental conditions using the maxima in the AFCs; these conditions comprised of four levels of sleep stage (REM, three stages of NREM), three levels of recording site $(\mathrm{Fz}, \mathrm{Cz}, \mathrm{Pz})$, and two levels of stimulus type (deviant, standard). Accordingly, there were altogether 24 experimental conditions.

Figs. 2 and 3 show how the gamma band activity in the early poststimulus time window behaves in different stages of sleep (REM and three stages of NREM sleep), recording sites (Fz, Cz, $\mathrm{Pz}$ ), and stimulus types (deviant, standard). As in the AFCs, gamma enhancement relative to the prestimulus baseline was specifically obtained in REM. For the NREM stages of sleep, digital filtering could not form the bases for conclusive 
statements. Early poststimulus gamma activity was obtained from $\mathrm{Fz}$ in Stage 2 in response to the deviant and standard stimuli (Figs. 2 and 3). However, those at the central recording sites could not be distinguished from the gamma bursts that occur in the prestimulus interval and from the gamma activity in the late poststimulus interval. There might be a gamma-band activity in Stage 3 to especially the standard stimuli and in Stage 4 to the deviant stimuli. However, in both stages, the enhancement was too low to allow for any clear statement.

\subsection{Predictability of N1 from oscillatory activity in the various frequency ranges}

The present study found that the maxima in the lower frequency ranges included the delta and the theta responses (Fig. 1). The predictability of N1 peak amplitude from the amplitudes of the delta, theta and higher frequency oscillations that also included the gamma-band activity was studied in REM and the three stages of NREM sleep using stepwise multiple regression analysis. The predictors were the amplitudes of the delta, theta and higher frequency oscillations at the latencies of the N1 peak. The predicted variable was the amplitude on the actual ERP waveform that coincided with the N1 peak. This analysis thus demonstrates the degree to which the amplitude of the N1 peak can be reconstructed from the amplitudes of the oscillatory responses that contribute to the amplitude of N1. Previous analysis had not shown significant differences between the N1 amplitudes at the different recording sites. Stepwise regression analyses were thus conducted on one of the recording sites; this site was chosen as the vertex.

Using the stepwise method, significant models emerged for all stages of sleep. The parameters for Stage 2 were $F(4,12)$ $=604.660, p=.0005$; those for Stage 3 were $F(3,8)=48.854$, $p=.0005$; those for Stage 4 were $F(3,8)=86.178, p=.0005$; those for REM were $F(3,8)=80.951, p=.0005$. All beta values were found significant (Table 1). For all stages of sleep, beta values showed that the amplitudes of the delta had the highest predictive power, followed by the amplitudes of the theta and

\section{Table 1}

Summary table for stepwise regression analyses with deviant-elicited ERP peak (N1) amplitudes as predicted variable and oscillations (delta, theta and higher frequencies) as predictor variables

\begin{tabular}{llllrl}
\hline & & $R^{2}$ & $\begin{array}{l}\text { Standardized } \\
\text { Beta }\end{array}$ & \multicolumn{1}{l}{$t$} & $p$ \\
\hline Stage 2 & Delta & 0.920 & 0.881 & 26.553 & 0.000 \\
& Theta & 0.984 & 0.281 & 11.460 & 0.000 \\
& Higher & 0.997 & 0.100 & 3.219 & 0.012 \\
Stage 3 & Delta & 0.530 & 0.992 & 8.983 & 0.000 \\
& Theta & 0.860 & 0.493 & 4.183 & 0.003 \\
& Higher & 0.948 & 0.350 & 3.701 & 0.006 \\
Stage 4 & Delta & 0.844 & 0.992 & 14.770 & 0.000 \\
& Theta & 0.924 & 0.258 & 3.833 & 0.005 \\
& Higher & 0.970 & 0.220 & 5.509 & 0.008 \\
REM & Delta & 0.803 & 0.902 & 12.909 & 0.000 \\
& Theta & 0.928 & 0.363 & 5.573 & 0.001 \\
& Higher & 0.968 & 0.217 & 3.191 & 0.013 \\
\hline
\end{tabular}

Recording site: Fz. then the higher frequencies. Accordingly, the major contributor to the ERP peak amplitude was the delta response.

The proportion of variance that the delta amplitude explained $\left(R^{2}\right)$ was between 0.530 and 0.920 ; the minimum was found for N1 under Stage 3 (the maximum was for N3 under Stage 3). The increase in the proportion of explained variance by addition of the theta amplitude to the regression equation was between 0.064 and 0.330; the maximum was for N1 under Stage 4 (the minimum was for N3 under Stage 3). The increase in the proportion of explained variance by addition of the amplitude of the higher frequencies to the regression equation was between 0.013 and 0.088; the maximum was for N1 under Stage 3 (the minimum was for N3 under Stage 4). Compared to the other peaks (P2, N2, P3, N3), N1 peak amplitude was thus maximally formed by the theta response and minimally by the delta response. The higher frequencies had the highest contribution to the amplitude of N1 peak.

\subsection{Time-frequency component analysis (TFCA)}

The presence of gamma response was determined in the present study using the automated gamma detection technique that was outlined in Section 2.4.4. This technique classified the poststimulus gamma band oscillations as gamma responses or as gamma rhythms that fail to meet the criteria for gamma responses. The proportions of the gamma responses in the three recording sites were found to be as follows: $F z=29 / 100$, $\mathrm{Cz}=33 / 100, \mathrm{Pz}=26 / 100$. The proportions for stages of sleep were found to be as follows: Stage $2=25 / 66$, Stage $3=7 / 66$, Stage $4=22 / 66, R E M=23 / 66$. Cochran test was used for evaluating the differences among the proportions of the gamma responses for recording sites and for sleep stages. The difference in the proportions of the gamma responses was not found significant for recording sites. The difference in the proportions of the gamma responses for the stages of sleep was found significant, $\chi^{2}=15.852, d f=3, p=.001$.

Figs. 4-6 show the results of the TFCA analyses that were performed for the gamma frequency range on data that were obtained from the $\mathrm{Fz}, \mathrm{Cz}$ and $\mathrm{Pz}$ recording sites, respectively. The frequency limits of the gamma components in Figs. 4-6 were studied for each combination of experimental conditions (four levels of stage, three levels of recording site and two levels of stimulus type) within the frequency range of $25-50 \mathrm{~Hz}$ (Karakaş and Başar, 1998). In these three figures, sleep stages included Stage 2 (panels 1-4), Stage 3 (panels 5-8), Stage 4 (panels 9-12) and REM (panels 13-16). For each stage, the upper panels represent the findings for the deviant stimuli (panels 1-2, 5-6, 9-10,13-14) and the lower panels represent the findings for the standard stimuli (panels $3-4,7-8,11-12$, 15-16).

The first columns in Figs. 4-6 show the composite timefrequency distributions of the components in the gamma range that were obtained by means of TFCA. Each panel was reconstructed using the individually extracted gamma components. The composite TFCA distributions showed that the ERP waveforms were composed of multiple gamma components that were localized in the time-frequency plane. Irrespective of 

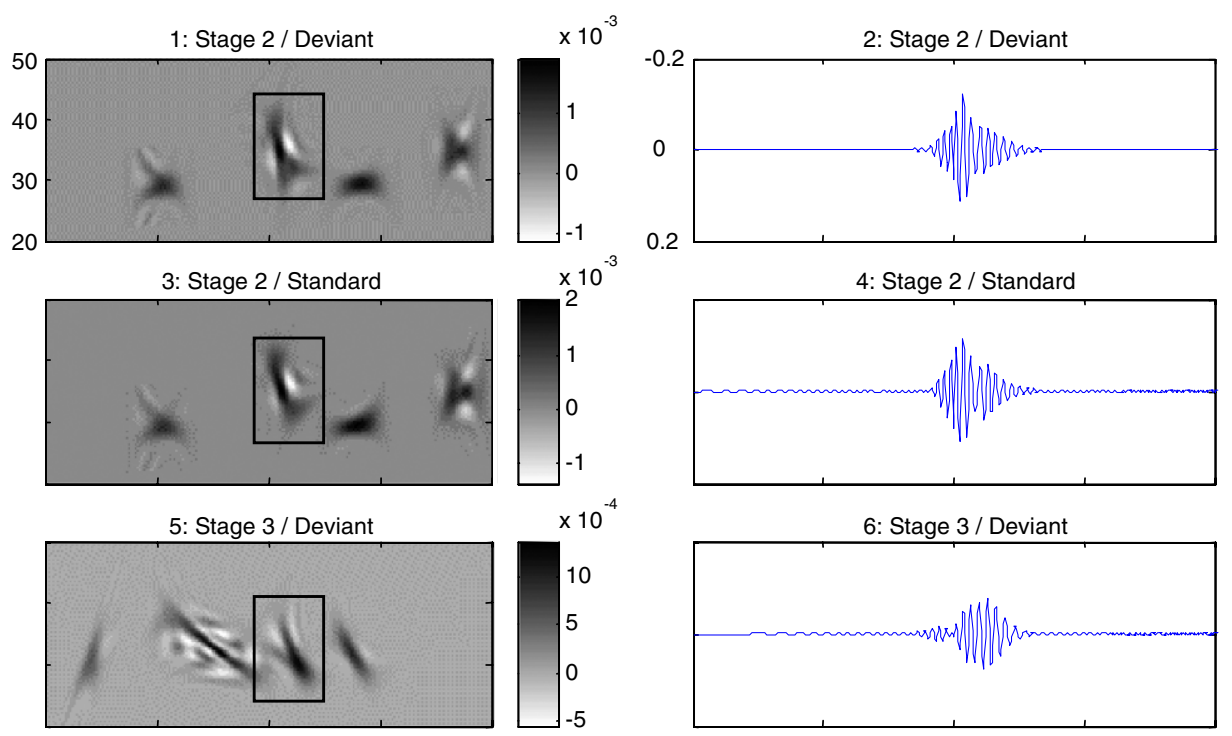

$\times 10^{-4}$<smiles>[Mg]</smiles>

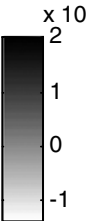

.

6: Stage 3 / Deviant

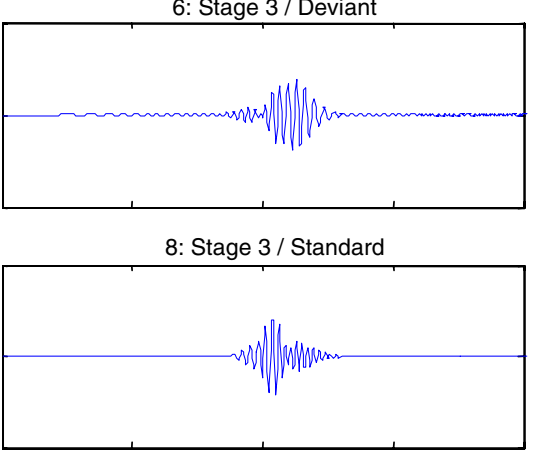

10: Stage 4 / Deviant

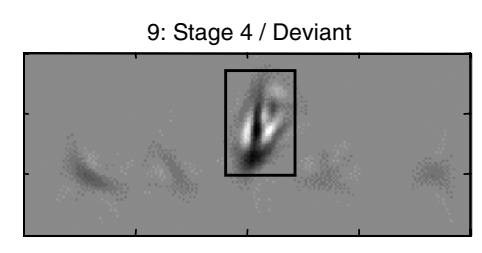

$\times 10^{-4}$

$\times 10^{-4}$
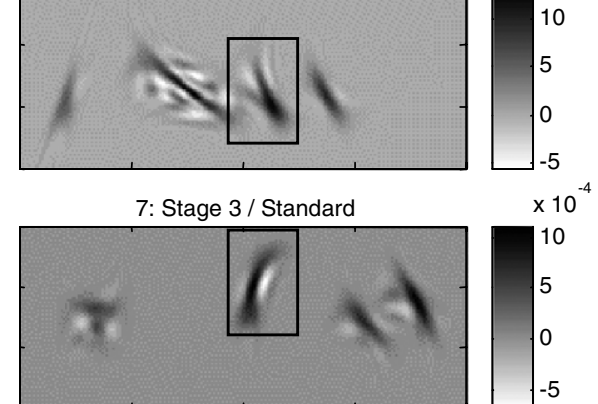

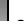
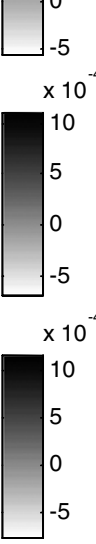

11: Stage 4 / Standard

$\times 10^{-4}$
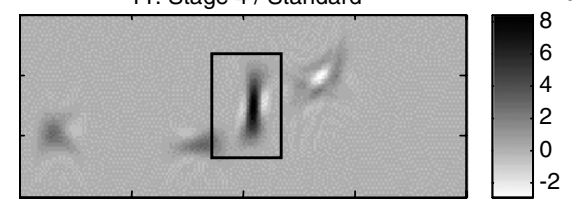

13: REM / Deviant

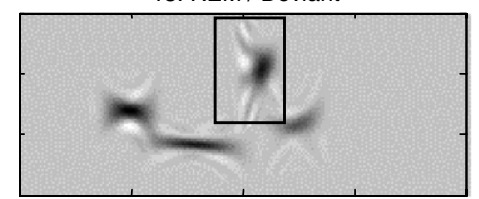

$\times 10^{-4}$

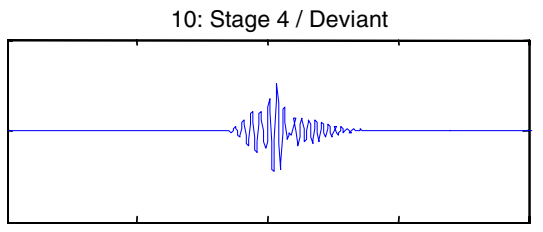

12: Stage 4 / Standard

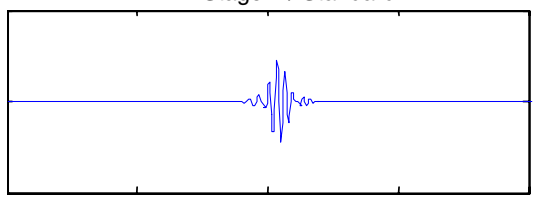

14: REM / Deviant
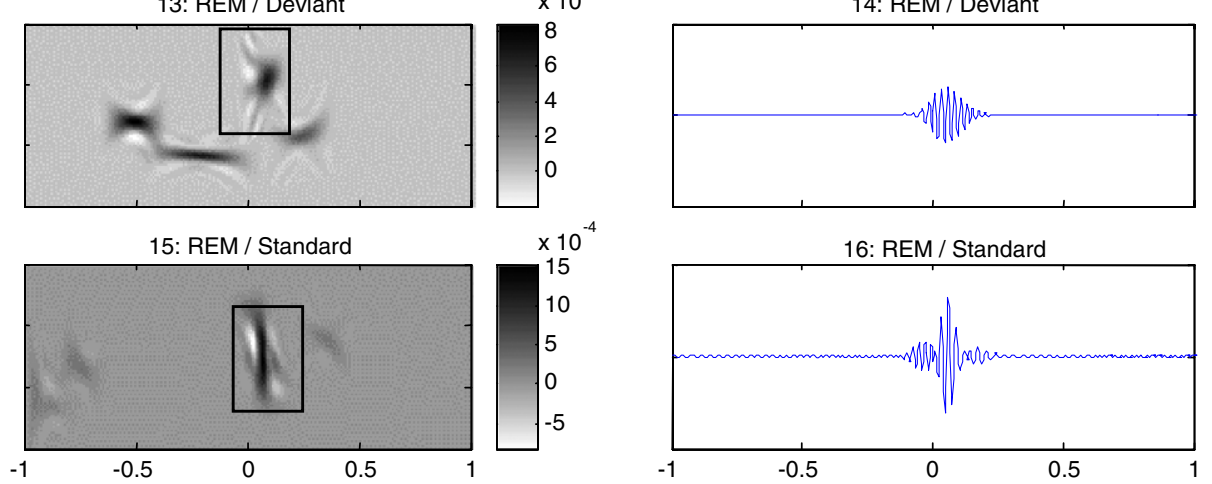

16: REM / Standard

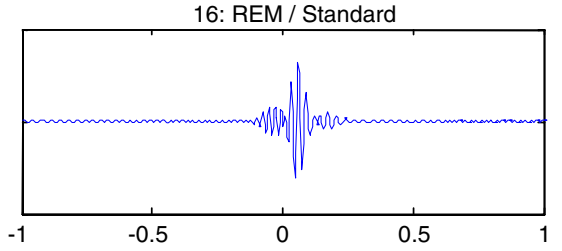

Fig. 4. The composite figure displays the results of TFCA analysis on frequencies in the gamma range. Recording site: Fz. Sleep stages include Stage 2 (panels 1-4), Stage 3 (panels 5-8), Stage 4 (panels 9-12) and REM (panels 13-16). For each sleep stage, the uppermost panels are for the deviant stimuli and the lowermost ones for the standard stimuli. First column: composite time-frequency representations of the gamma components that were extracted using TFCA. In each panel, the TFCA component, which corresponds to the early gamma response, is bounded in a rectangle. Second column: the time-domain signal of the extracted component. $Y$-axis (left): frequency in $\mathrm{Hz}$ (the axis at Stage 2 is valid for all time-frequency representations). $Y$-axes (right): intensity. Note that the individual time-frequency representations have scales proportional to the strength of the corresponding component. $X$-axes: time in seconds. 

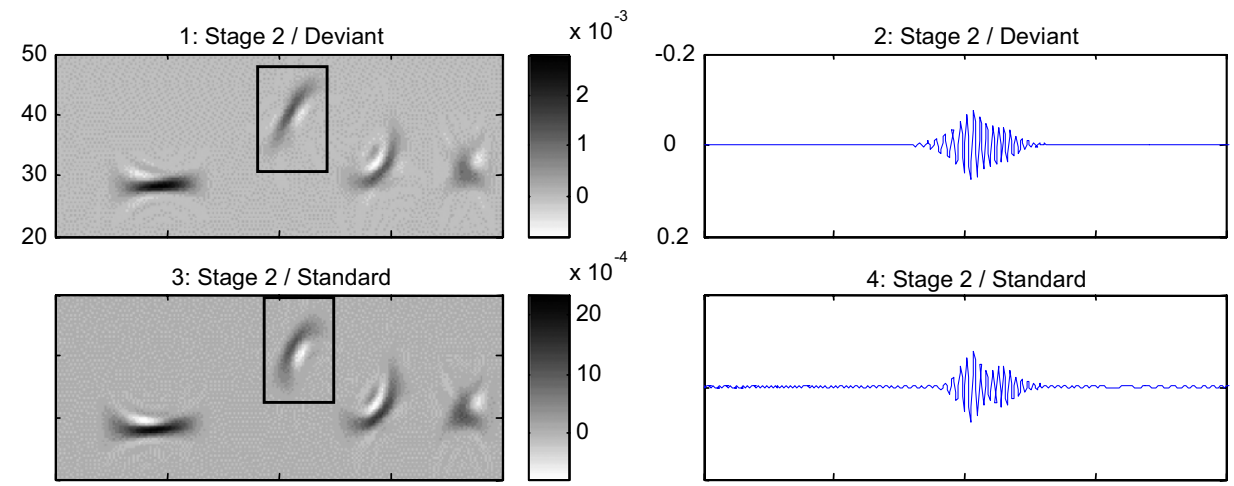

5: Stage 3 / Deviant $\times 10^{-4}$
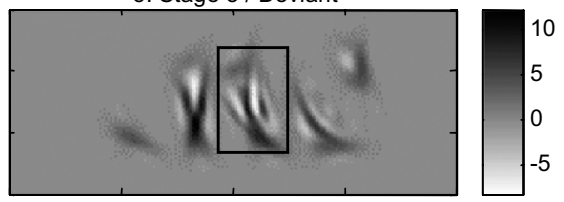

6: Stage 3 / Deviant

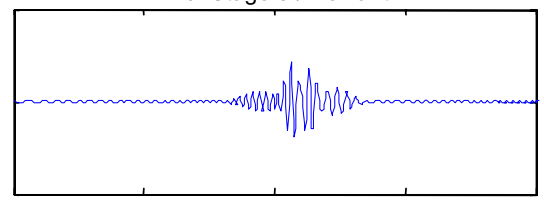

7: Stage 3 / Standard $\times 10^{-4}$
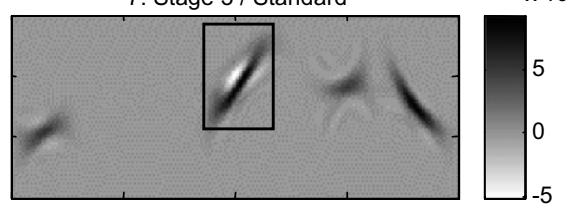

9: Stage 4 / Deviant $\times 10^{-4}$
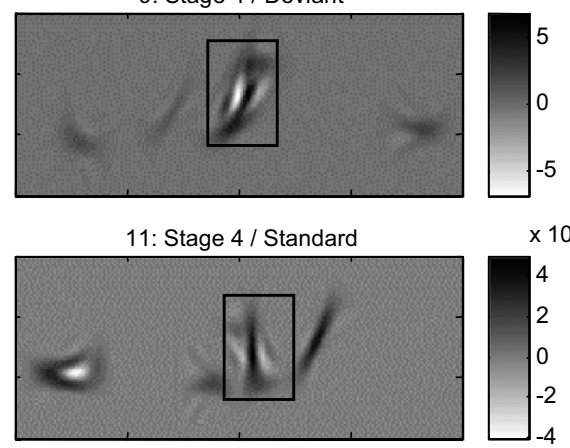

$\times 10^{-4}$

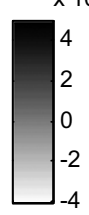

13: REM / Devian $\times 10^{-4}$
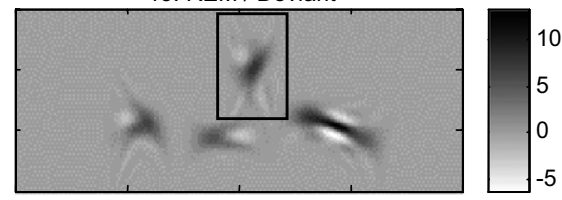

15: REM / Standard $\times 10^{-3}$
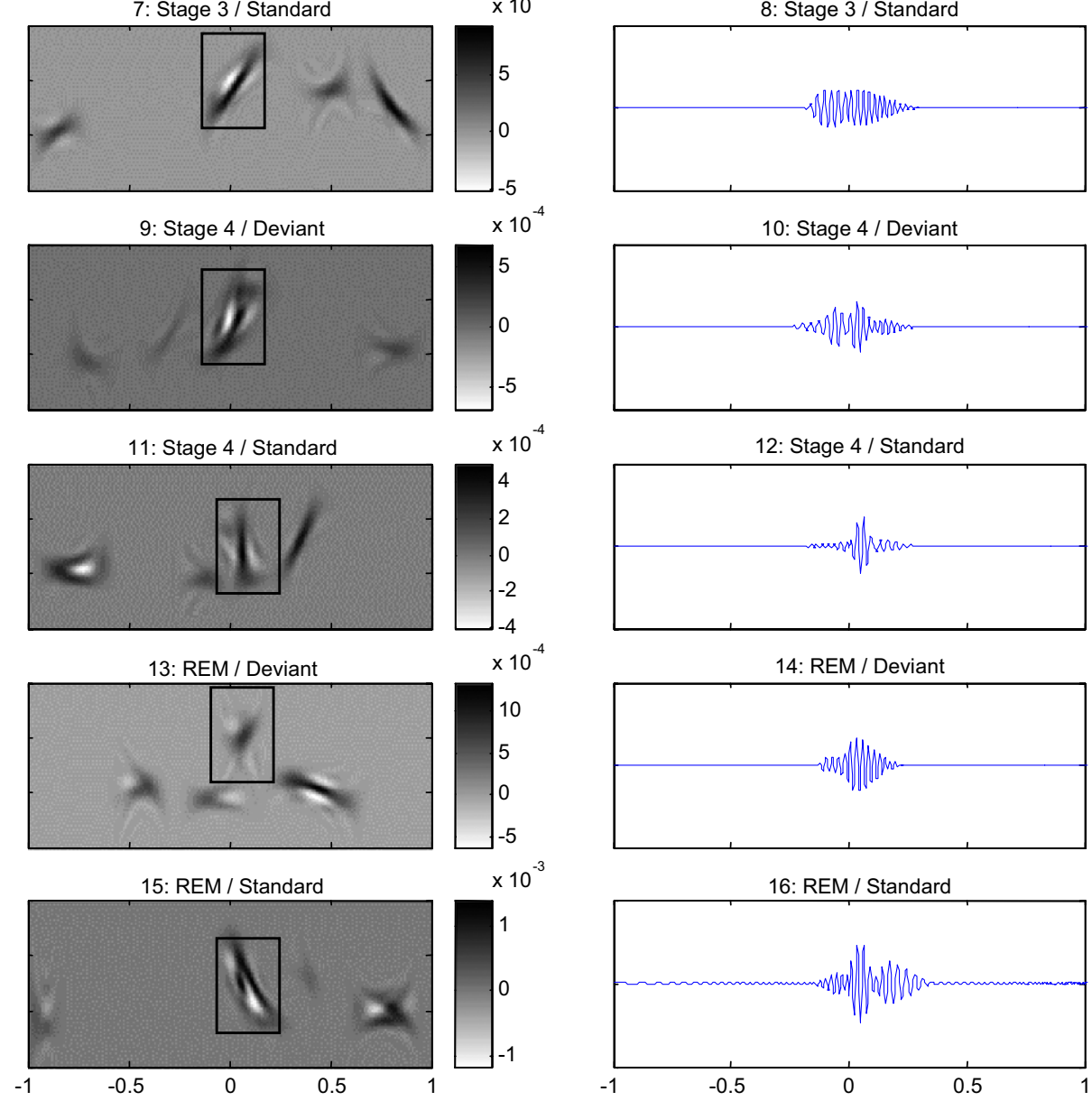

12: Stage 4 / Standard

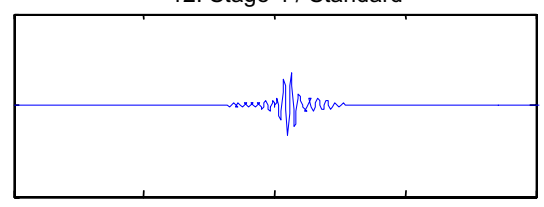

14: REM / Deviant

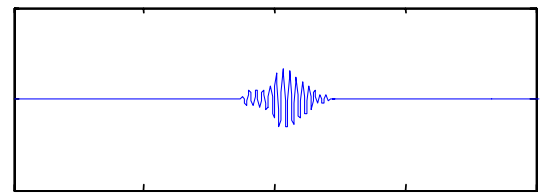

16: REM / Standard

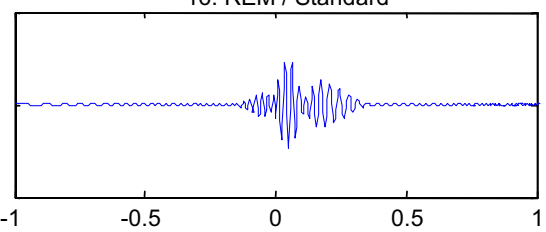

Fig. 5. The composite figure displays the results of TFCA analysis on frequencies in the gamma range. Recording site: Cz. Sleep stages include Stage 2 (panels 1-4), Stage 3 (panels 5-8), Stage 4 (panels 9-12) and REM (panels 13-16). For each sleep stage, the uppermost panels are for the deviant stimuli and the lowermost ones for the standard stimuli. First column: composite time-frequency representations of the gamma components that were extracted using TFCA. In each panel, the TFCA component, which corresponds to the early gamma response, is bounded in a rectangle. Second column: the time-domain signal of the extracted component. $Y$-axis (left): frequency in $\mathrm{Hz}$ (the axis at Stage 2 is valid for all time-frequency representations). $Y$-axes (right): intensity. Note that the individual time-frequency representations have scales proportional to the strength of the corresponding component. $X$-axes: time in seconds. 

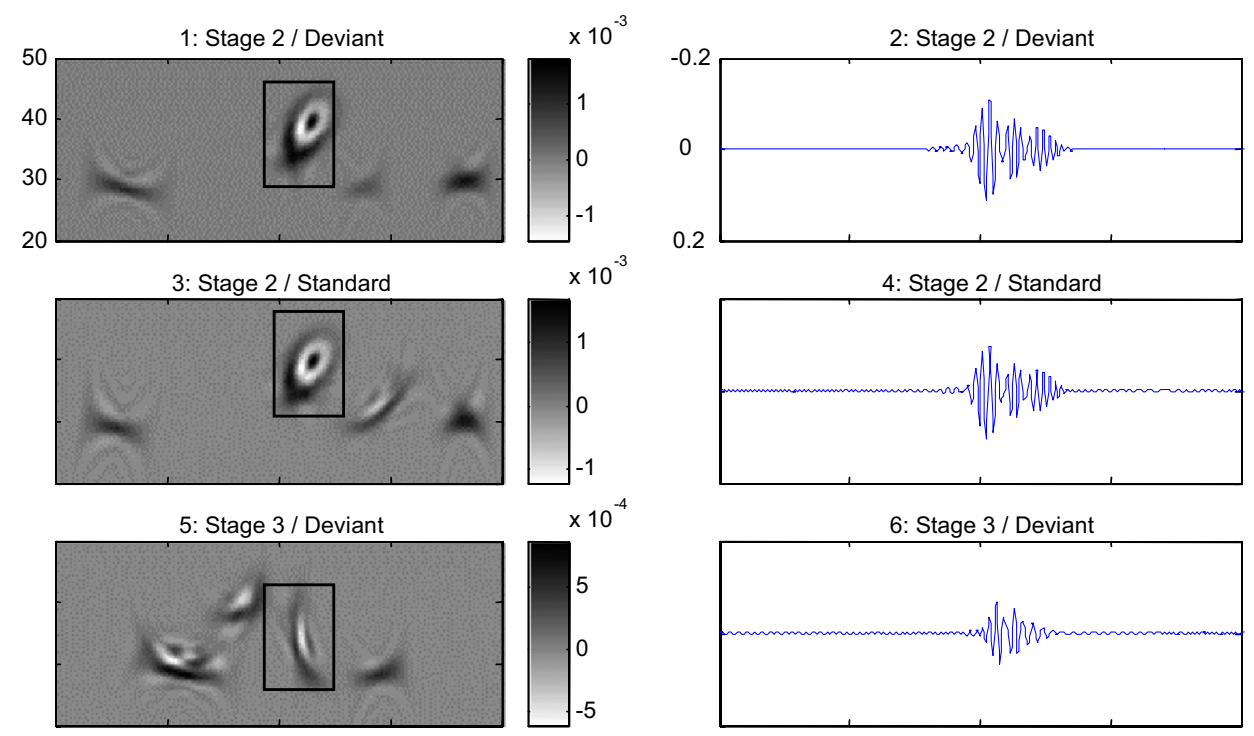

$\times 10^{-4}$

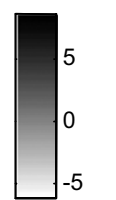

6: Stage 3 / Deviant

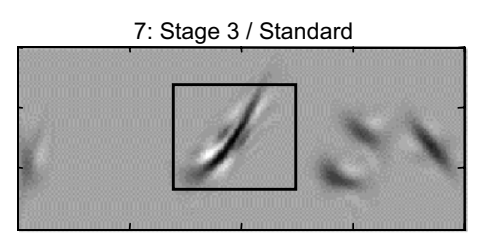

$\times 10^{-4}$
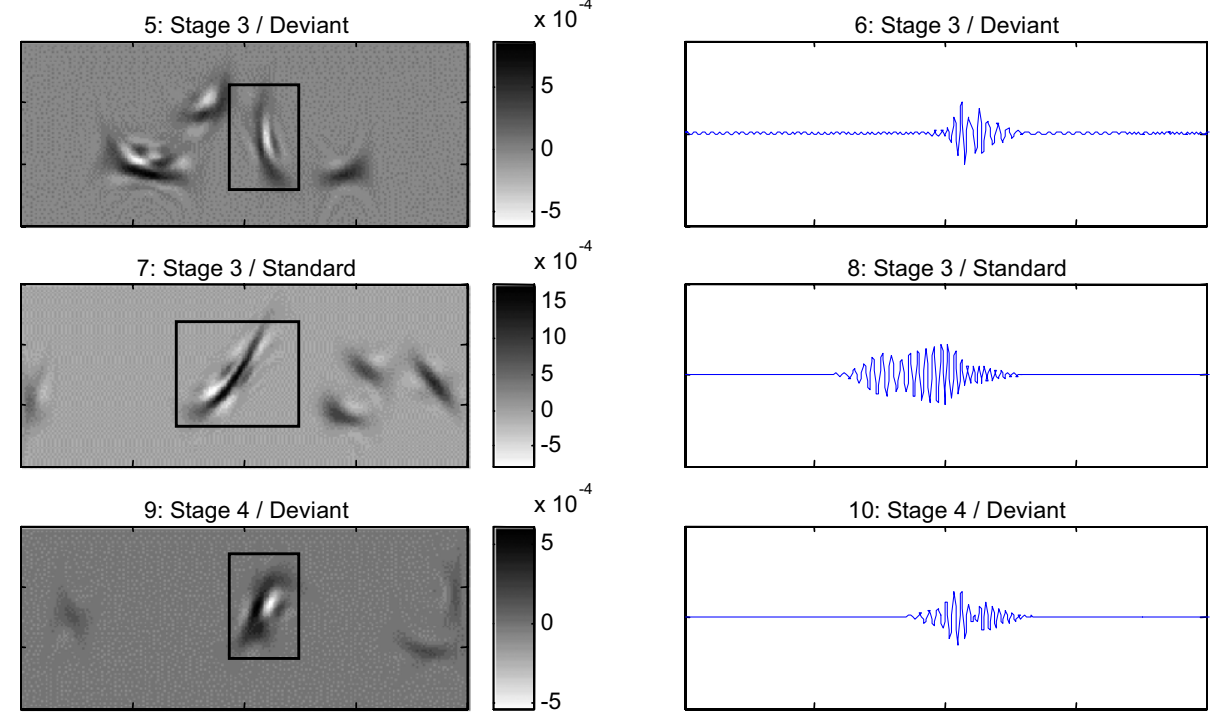

10: Stage 4 / Deviant
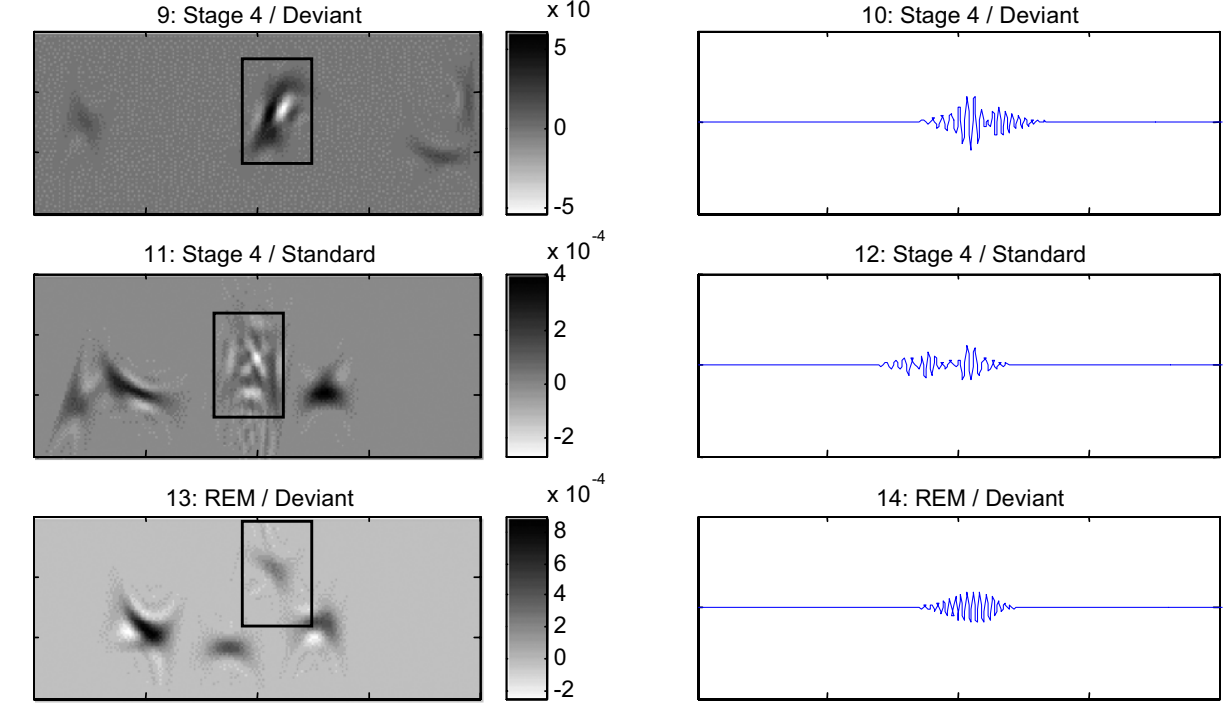

$\times 10^{-4}$

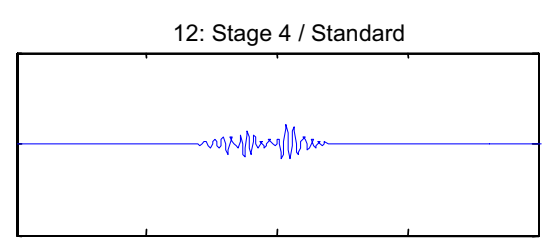

14: REM / Deviant

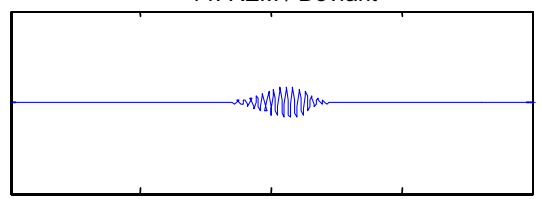

15: REM / Standard $\times 10^{-4}$
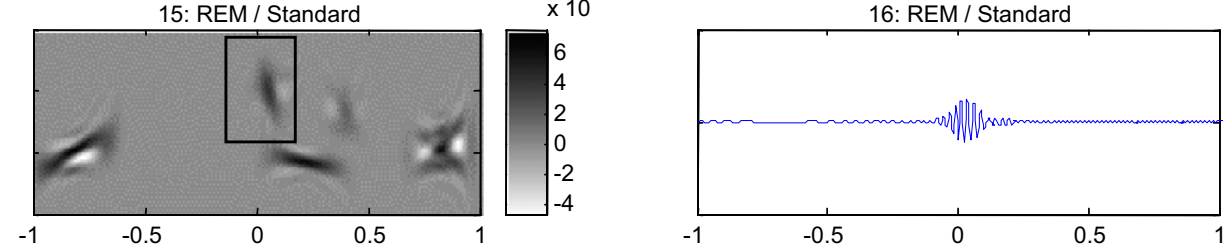

Fig. 6. The composite figure displays the results of TFCA analysis on frequencies in the gamma range. Recording site: Pz. Sleep stages include Stage 2 (panels 1-4), Stage 3 (panels 5-8), Stage 4 (panels 9-12) and REM (panels 13-16). For each sleep stage, the uppermost panels are for the deviant stimuli and the lowermost ones for the standard stimuli. First column: composite time-frequency representations of the gamma components that were extracted using TFCA. In each panel, the TFCA component, which corresponds to the early gamma response, is bounded in a rectangle. Second column: the time-domain signal of the extracted component. $Y$-axis (left): frequency in $\mathrm{Hz}$ (the axis at Stage 2 is valid for all time-frequency representations). $Y$-axes (right): intensity. Note that the individual time-frequency representations have scales proportional to the strength of the corresponding component. $X$-axes: time in seconds. 
recording site and stimulus type, these components existed in both the prestimulus and poststimulus epochs.

The first columns in Figs. 4-6 also show the TFCA components (Component 1) that correspond to the early gamma activity; these components were bounded in rectangles. With respect to the prestimulus baseline, the time signals showed enhancement upon stimulation in all stages of sleep, at all recording sites and in response to both the deviant and the standard stimuli. TFCA shows that the early gamma component occurred within a frequency range of $25.15 \mathrm{~Hz}$ and $49.00 \mathrm{~Hz}$. The time window extended to $272 \mathrm{~ms}$ for the deviant stimuli and to $284 \mathrm{~ms}$ for standard stimuli.

The effects of sleep stage (REM and NREM Stages 2, 3 and 4), recording site $(\mathrm{Fz}, \mathrm{Cz}, \mathrm{Pz}, \mathrm{F} 3, \mathrm{~F} 4, \mathrm{P} 3, \mathrm{P} 4)$ and stimulus type (deviant, standard) were analyzed using $4 \times 7 \times 2$ univariate ANOVA with repeated measures on all factors (Vasey and Thayer, 1987). The dependent variables were three parameters of the TFCA. As is required by ANOVA, the dependent variables comprised of the ratio scale. Data that violated the assumption of normality were converted to the normal distribution using log transformation. Analyses were then performed on $z$-transformed data. Equality of variances was checked for sphericity using Mauchly's test. If data were not found to be spherical, revised degrees of freedom were obtained by multiplying the original values by the Greenhouse-Geisser epsilon values. The significance levels of the $F$-values were obtained using these degrees of freedom.

Analyses were performed on the three parameters of TFCA separately. One of the parameters was the maximum value of the time-frequency distribution where the extracted gamma component reached its highest value (Figs. 4-6, column 1). The results showed that none of the variables had a significant effect on the maximum value of the time-frequency distribution of the extracted TFCA component. TFCA provided not only time-frequency distributions but also descriptions on the timedomain characteristics of extracted components (Figs. 4-6, column 2). The second parameter was related to the time domain signal of the extracted TFCA components. It represented the energy of the extracted component and was the integral (sum) of the magnitude square of the time-domain description of the extracted component. Again, none of the effects were significant, showing that the energy of the time domain signal of the extracted components did not vary according to sleep stage, recording site or stimulus type. The third parameter was also related to the time domain signal of the extracted component. This parameter indicated the maximum magnitude of the time domain signal of the extracted TFCA component. Again, none of the effects were significant showing that the degree of poststimulus gamma enhancement did not vary according to sleep stage, recording site or stimulus type. Accordingly, when the gamma band activity was studied in the time-frequency domain by the recently developed technique of TFCA (Özdemir et al., 2005), the maximum value of the timefrequency representation of the extracted gamma component, the energy of the time domain signal that correspond to the extracted component, and the maximum magnitude of this component was similarly obtained in different stages of sleep, recording sites and stimuli.

\section{Discussion}

The early, time-locked gamma response (approximately $150 \mathrm{~ms}$ poststimulus) is related to sensory-perceptual processing in wakefulness (for reviews, see Başar-Eroğlu et al., 1999; Galambos, 1992; Karakaş et al., 2001). The present study used the early gamma response as a tool for investigating brain's responsiveness during stages of sleep to exogenous stimuli. The response was obtained under an auditory cognitive paradigm (OB-p) during REM and NREM stages of sleep in the natural overnight sleep of young, healthy, male participants.

\subsection{The distribution of the gamma band response to stages of sleep and recording sites}

The present study was focused on whether stimulation evoked a gamma response, in other words, whether gamma parameters (e.g., amplitude, energy) were enhanced upon stimulation. Such studies are usually faced with the problem of component extraction since when compared with lower frequency components such ones as the delta and theta oscillations, the gamma oscillation has markedly reduced amplitude. As a first approach, the present study used methods of frequency domain, which assume that the studied system is linear and stationary (Özdemir et al., 2005). Amplitude frequency characteristics showed that the gamma band activity was specifically a characteristic of the REM stage and was observed upon both the deviant and the standard stimuli (Fig. 1). This result was obtained with also the response-adaptive digital filtering (Figs. 2 and 3). In the other stages, however, conclusive statements could not be possible since either the response could either not be distinguished from the prestimulus or the poststimulus gamma bursts that occurred later in the recording time or stimulus-elicited enhancement was too low to render definite statements.

Occurring in a specific time window, the time-locked gamma response is a time-varying, nonstationary signal. Being an oscillation, it should be represented in the time and frequency planes simultaneously. The present study thus used the recently introduced technique of time-frequency component analysis (TFCA) (Özdemir et al., 2005; Karakaş et al., in press) as the third technique for studying the early poststimulus gamma activity. TFCA showed that the gamma component occurred not only in REM but also in NREM stages of sleep. The reason why the EEG gamma did not reset in REM upon stimulation (Llinas and Ribary, 1992, 1993) and the failure to demonstrate it in NREM stages of sleep may possibly have been due to the utilized technique of component extraction (Fourier analysis and digital filtering) which assumes system linearity and stationarity and also due to the limitation of the domain that is analyzed (frequency-domain only).

According to results on analysis of variance, the recording site affected none of the parameters of TFCA (maximum value of the extracted gamma component on the time-frequency 
plane, the maximum magnitude of the time signal that corresponds to the TFCA component, the energy of this component). Similarly, according to the results of Cochran test, the proportions of the gamma responses that were determined by the automated gamma detection technique were found to be similarly distributed over the studied recording sites. These findings show that the early gamma component was similarly obtained over the studied topographical sites.

In wakefulness, on the other hand, the gamma activity in the early poststimulus period was obtained from the frontocentral recording sites (Karakaş and Başar, 1998). Sleep is characterized with synchrony between different structures/topographical sites of the brain. This well-known phenomenon in sleep EEG distinguishes sleep from the wakeful activation in the brain. The present study showed that the high synchrony between the studied topographical sites also held for the gamma component. Winter et al. (1995) argued that a totally different system is responsible for the processing of stimuli during sleep. With transition from the waking to the sleep system, changes occurred in the scalp distribution of the components. The same change of topography was also observed by Feige and colleagues (1997). Using a CNV-type paradigm, it was found that in Stage 2, slow ERPs changed from a CNV-type frontocentral negativity to a central positivity. In REM, the component was obtained as a centroparietal negativity. These topographical changes suggest that brain areas involved in processing auditory stimuli change from waking to sleep. Further research that would involve detailed study of topographical variations in especially the event-related oscillations is necessary to unravel this dynamic transition in sleep.

The binding hypothesis (Gray and Singer, 1989; Joliot et al., 1994) uses gamma activity as the mechanism of feature linking in perception. Although the original studies found a widespread distribution of the gamma activity that allowed for the binding of distant brain regions (Llinas and Ribary, 1993), other studies on brain electrophysiology or brain imaging failed to find longdistance coherence or simultaneous activation (Menon et al., 1996; Steraide and Amzica, 1996). The present study did not investigate coherences but did show that early gamma component did not differentiate between recording sites. This finding suggests that the binding hypothesis that uses the gamma response may be plausible. Future work should be devoted to a detailed coherence analysis of topographical regions for testing this hypothesis once again, using an appropriate technique which would allow analysis in the time-frequency domain.

\subsection{Possible cognitive correlates of the early gamma response in sleep}

In wakefulness, the early, time-locked gamma response was found to occur over a spectrum of cognitive paradigms and upon both the deviant and standard stimuli of the active and passive oddball paradigm (Karakaş and Başar, 1998). The conclusion of this and other similar studies in the literature was that the early gamma response represents sensory/perceptual processing (for reviews, see Galambos, 1992; Gurtubay et al.,
2001; Karakaş et al., 2001; Karakaş and Başar, 1998). In the present study sleep stage, recording site and stimulus type did not affect parameters of TFCA, which consisted of the maximum value of the time-frequency representation of the extracted gamma component, the energy of the time domain signal of the extracted gamma component and the maximum magnitude of this component. A general statement would thus be that stimulation produces an early gamma component regardless of sleep stage and nature of the stimulus over the studied topographical sites.

In the absence of an ample amount of studies where the cognitive meaning of the gamma band response was investigated, an approach for understanding its cognitive meaning may be to consider the components that co-occur with it. In the window where the early gamma response occurs, there is the N1 ERP component in sleep. The present study found that N1 did not vary according to sleep stage or stimulus type; it was thus not functional in differentiating the deviant from the standard stimuli or differentiating between stages of sleep. It was with such a processing that the gamma component co-occurs.

In the literature, sleep N1 was obtained irrespective of stimulus type and/or sleep stage (Campbell and Colrain, 2002; Winter et al., 1995). Being induced by sensory stimulation of all types, N100-P200 was considered an index for the presence (or absence) of sensory stimulation (Kevanishvili and Specht, 1979; Weitzman and Kremen, 1965). Sleep N1 was associated with sensory sensitivity and the arousal factor of the orienting response (Atienza et al., 2001). The amplitude of the N1 was found to diminish throughout Stage 1, 2 and 4 (for a review, see Campbell, 2002); this was interpreted as the loss of attentional capacity with sleep. Thus, the time window where the early gamma response also occurs is devoted, according to these findings, to early stimulus processing that also involves sensory processes.

The present study found that the sleep N1 was critically dependent on the early theta response. Stepwise regression analysis showed that, in sleep, the first cycle of the theta response contributed maximally to the amplitude of $\mathrm{N} 1$; this oscillation also contributes maximally to the amplitude of wakefulness N100 (Karakaș et al., 2000). The early theta response represents anticipation and preferential detection of selected stimuli and a complex set of cognitive processes whereby selective attention becomes focused on a task-relevant template (Karakaş et al., 2000; Schürmann et al., 1995). Such functions are closely related to the sensory register (a specific information is selected amongst many others) and perception (selection is based on a given template or percept) (Eysenck, 2001; Karakaş and Başar, in press).

Shortly, early gamma response occurs regardless of state of consciousness (REM and stages of NREM sleep) and stimulus type. The activity co-occurs with $\mathrm{N} 1$ and the early theta response that contribute to its amplitude. According to the specified literature, both $\mathrm{N} 1$ and early theta response is related to sensory/perceptual processing. Altogether, these findings suggest that the early gamma response in sleep also represents sensory/perceptual operations. These conclusions are valid, however, within the conditions of the present study: The 
stimulation was delivered in the present study over loudspeakers, and analyses were conducted on sweeps that were recorded throughout the total duration of the first night sleep in the laboratory. A test on the effects of these limiting conditions should be the task of future research. This study was conducted on a subset of phenomena of Stage 2 that comprised of the spindles. Another area for future research should be the investigation of the gamma response in epochs with $\mathrm{K}$ complexes.

Studies in the literature concluded that the activated state in REM is limited to sensory processing. Llinas et al. (Llinas and Ribary, 1993; Joliot et al., 1994) found that the thalamocortical system was accessible to sensory input in both waking and REM. Topological studies found that activation in REM was mainly in the modality-specific areas. Sensory processing in REM could develop into perceptual processing, however, if task conditions so require. That the early gamma response in sleep is related to sensory/perceptual operations was suggested by findings of the present study on the REM stage. REM stage was found to be characterized by the earlier components of stimulus processing that included the early gamma response, the early theta response and N1. In REM, the components of extended processing which included the late theta (second cycle) that contributed to $\mathrm{N} 2$ and $\mathrm{P} 3$ and the delta oscillation that contributed to specifically N3 did not exist (Figs. 2 and 3). According to these findings, selective processing of auditory stimuli terminated in REM after an early processing. According to the sensory-inhibition theory, theta is associated with the generalized inhibition of non-relevant sensory systems during perceptual processing (Kamarajan et al., 2004; Sainsbury, 1998). A deduction from this theory would be that further processing of the stimulus is inhibited in REM, possibly in favor of processing the internal stimuli that emanate from the dreams.

Being related to sensory/perceptual operations, the early poststimulus gamma activity basically represents bottom-up processing; through bottom-up processing, the early processing systems determine what enters the system. However, higher order systems exert an effect on what and how information will be processed and this is top-down processing (Engel et al., 2001). Perception itself is a case of both bottom-up and topdown processing that involves an interactive process between the sensory register and the long-term memory (Karakaş and Başar, in press). Accordingly, gamma activity in wakefulness was found to be related to also top-down processing. Early gamma demonstrated individual differences and this was not related to gender differences (Karakaş et al., in press). Gamma activity was also found to be correlated with neuropsychological test scores (Karakaş et al., 2003). The suppression of the early gamma band response in the frontal areas indicated a deficient top-down processing mechanism (Padmanabhapillai et al., 2005). The relationship of these two basic processes was explained through neuronal synchronization between attentional control structures and areas for stimulus representation (Fell et al., 2003). Accordingly, although it is specifically related to sensory processing, the gamma response of sleep may also be a representative of complex cognitive operations that derive from the top-down processes of the brain.
To summarize, in sleep, the brain responded to auditory stimuli of the passive oddball paradigm with an early gamma response. Gamma response co-occurred with the first cycle of the theta response and N1. These findings render the conclusion that the gamma response in sleep represents, as in wakefulness, sensory/perceptual processing. Gamma response was obtained in REM, all stages of NREM sleep and wakefulness to both the deviant and standard stimuli. The implication of these findings is that the sensory and possibly the perceptual processing that the gamma response represents are similarly obtained under all stages of sleep. Some stages of sleep, e.g., REM, terminate with an early processing. Processing in even such a stage is not as simple as it seems, considering the top-down influences on the early gamma response and the modifications that they exercise on cognitive processing. In other stages of sleep, processing of the stimuli continued. The late negativity in Stage 2 and the delta oscillation that contributed to it is a demonstration of the extent to which stimulus processing may occur in sleep.

\section{References}

Atienza, M., Cantero, J.L., Gomez, C.M., 2001. The initial orienting response during human REM sleep as revealed by the N1 component of auditory event-related potentials. Int. J. Psychophysiol. 41, 131-141.

Başar, E., 2004. Memory and Brain Dynamics: Oscillations Integrating Attention, Perception, Learning and Remembering. CRC Pr, London.

Başar-Eroğlu, C., Strüber, D., Kruse, P., Başar, E., Stadler, M., 1996. Frontal gamma-band enhancement during multistable visual perception. Int. J. Psychophysiol. 24, 113-125.

Başar-Eroğlu, C., Başar, E., Schürmann, M., Schütt, A., Strüber, D., Stadler, M., Karakaş, S., 1999. Gamma-band responses in the brain: functional significance (Ch. 26). In: Başar, E. (Ed.), Brain Function and Oscillations: II. Integrative Brain Function. Neurophysiology and Cognitive Processes. Springer-Verlag, Heidelberg.

Bressler, S.L., 1990. The gamma wave: a cortical information carrier. Trends Neurosci. 13, 161-162.

Bressler, S.L., Tognoli, E., in press. Operational principles of neurocognitive networks. Int. J Psychophysiol (Special Issue on Models and Theories of Brain Function with Special Emphasis on Cognitive Processing, Guest Editors: S. Karakaş, E. Bașar).

Brualla, J., Romero, M.F., Serrano, M., Valdizan, J.R., 1998. Auditory eventrelated potentials to semantic priming during sleep. Electroencephalogr. Clin. Neurophysiol. 108, 283-290.

Burton, S.A., Harsh, J.R., Badia, P., 1988. Cognitive activity in sleep and responsiveness to external stimuli. Sleep 11, 61-68.

Campbell, K.B. (Guest Ed.), 2002. Event-Related Potential Measures of Information Processing During Sleep. Int. J Psychophysiol. 46(3), 159-286.

Campbell, K.B., Colrain, I.M., 2002. Event-related potential measures of the inhibition of information processing: II. The sleep onset period. Int. J. Psychophysiol. 46, 197-214.

Cote, K.A., Campbell, K.B., 1999. P300 to high intensity stimuli during REM sleep. Clin. Neurophysiol. 110, 1345-1350.

De Pascalis, V., Cacace, I., 2005. Pain perception, obstructive imagery and phase-ordered gamma oscillations. Int. J Psychophysiol. 56 (2), 157-169.

Engel, A., Fries, P., Singer, W., 2001. Dynamic predictions: oscillations and synchrony in top-down processing. Nat. Rev., Neurosci. 2, 704-716.

Eysenck, M.W., 2001. Principle of Cognitive Psychology, 2nd ed. Psychology Pr., New York.

Farwell, L.A., Martinerie, J.M., Bashore, T.R., Rapp, P.E., Goddard, P.H., 1993 Optimal digital filters for long-latency components of the event-related brain potential. Psychophysiology 30, 306-315.

Feige, B., Krieger, S., Lis, S., Jacobs, A., Kiemen, F., Hohagen, F., Berger, M., 1997. Auditory information processing in sleep. Proceedings of the 14th Int. 
Congress of EEG and Clinical Neurophysiology, Florence. Electroencephalogr. Clin. Neurophysiol. 103 (1), $72-73$.

Fell, J., Fernandez, G., Klaver, P., Elger, C.E., Fries, P., 2003. Is synchronized neuronal gamma activity relevant for selective attention? Brain Res. Brain Res. Rev. 42, 265-272.

Freeman, W., in press. A cinematographic hypothesis of cortical dynamics in perception. Int. J Psychophysiol (Special Issue on Models and Theories of Brain Function with Special Emphasis on Cognitive Processing, Guest Editors: S. Karakaș, E. Başar).

Fuster, J.M., 1995. Memory in the Cerebral Cortex: An Empirical Approach to the Neural Networks in the Human and Nonhuman Primate. MIT Press, Cambridge MA.

Fuster, J., in press. The cognit: a network model of cortical representation. Int. J Psychophysiol (Special Issue on Models and Theories of Brain Function with Special Emphasis on Cognitive Processing, Guest Editors: S. Karakaş, E. Başar).

Galambos, R., 1992. A comparison of certain gamma band $(40 \mathrm{~Hz})$ brain rhythms in cat and man. In: Başar, E., Bullock, T.H. (Eds.), Induced Rhythms in the Brain. Birkhaüser, Boston.

Gray, C.M., Singer, W., 1989. Stimulus-specific neuronal oscillations in orientation columns of cat visual cortex. Proc. Natl. Acad. Sci. U. S. A. 86, $1698-1702$

Gurtubay, A.M., Labura, A., Malanda, A., Iriarte, J., Artieda, J., 2001. Gamma band activity in an auditory oddball paradigm studied with the wavelet transform. Clin. Neurophysiol. 112, 1219-1228.

Gurtubay, I.G., Alegre, M., Labarga, A., Malanda, A., Artieda, J., 2004. Gamma band responses to target and non-target auditory stimuli in humans. Neurosci. Lett. 367 (1), 6-9.

Haig, A.R., Gordon, E., De Pascalis, V., Meares, R.A., Bahramali, H., Harris, A., 2000. Gamma activity in schizophrenia: evidence of impaired network binding? Clin. Neurophysiol. 111 (8), 1461-1468.

Herrmann, C.S., Mecklinger, A., 2001. Gamma activity in human EEG is related to high-speed memory comparison during object selective attention. Vis. Cogn. 8 (3-5), 593-608.

Herrmann, C.S., Mecklinger, A., Pfeifer, E., 1999. Gamma responses and ERPs in visual classification task. Clin. Neurophysiol. 110, 636-642.

Jokeit, H., Makeig, S., 1994. Different event-related patterns of $\chi$-band power in brain waves of fast- and slow-reacting subjects. Proc. Natl. Acad. Sci. U. S. A. 9, 6339-6343.

Joliot, M., Ribary, U., Llinas, R., 1994. Human oscillatory brain activity near $40 \mathrm{~Hz}$ coexists with temporal cognitive binding. Proc. Natl. Acad. Sci. U. S. A. $91,11748-11751$.

Kamarajan, C., Porjesz, B., Jones, K.A., Choi, K., Chorlian, D.B., Padmanabhapillai, A., Rangaswamy, M., Stimus, A.T., Begleiter, H., 2004. The role of brain oscillations as functional correlates of cognitive systems: a study of frontal inhibitory control in alcoholism. Int. J. Psychophysiol. 51, $155-180$.

Karakaş, S., Başar, E., 1998. Early gamma response is sensory in origin. A conclusion based on cross-comparison of results from multiple experimental paradigms. Int. J. Psychophysiol. 31 (1), 13-31.

Karakaş, S., Başar, E., in press. Models and theories of brain function in cognition within a framework of behavioral cognitive psychology Int. J Psychophysiol (Special Issue on Models and Theories of Brain Function with Special Emphasis on Cognitive Processing, Guest Editors: S. Karakaş, E. Basar).

Karakaş, S., Erzengin, Ö.U., Başar, E., 2000. A new strategy involving multiple cognitive paradigms demonstrates that ERP components are determined by the superposition of oscillatory responses. Clin. Neurophysiol. 111, $1719-1732$

Karakaş, S., Başar-Eroğlu, C., Özesmi, Ç., Kafadar, H., Erzengin, Ö.U., 2001. Gamma response of the brain: a multifunctional oscillation that represents a bottom-up with top-down processing. Int. J. Psychophysiol. 39 (2-3), $137-150$.

Karakaş, S., Bekçi, B., Erzengin, Ö.U., 2003. Early gamma response in human neuroelectric activity is correlated with neuropsychological test scores. Neurosci. Lett. 340, 37-40.
Karakas, S., Tüfekçi, İ., Bekçi, B., Cakmak, E.D., Doğutepe, E., Erzengin, Ö.U., Özkan, A., Arkkan, O., in press. Early time-locked gamma response and gender specificity. Int. J Psychophysiol.

Kevanishvili, Z.S., Specht, V., 1979. Human slow auditory evoked potentials during natural sleep and drug induced sleep. Electroencephalogr. Clin. Neurophysiol. 47, 280-288.

Lachaux, J.P., Rodriguez, E., Martinerie, J., Adam, C., Hasboun, D., Varela, F.J., 2000. A quantitative study of gamma-band activity in human intracranial recordings triggered by visual stimuli. Eur. J. Neurosci. 12 (7), 2608-2622.

Llinas, R.R., Pare, D., 1991. Of dreaming and wakefulness. Neuroscience 44, 521-535.

Llinas, R.R., Ribary, U., 1992. Rostrocaudal scan in the human brain: a global characteristic of the $40 \mathrm{~Hz}$ response during sensory input. In: Başar, E., Bullock, T.H. (Eds.), Induced Rhythms in the Brain. Birkhäuser, Boston.

Llinas, R., Ribary, U., 1993. Coherent 40-Hz oscillation characterizes dream state in humans. Proc. Natl. Acad. Sci. Neurobiol. 90, 2078-2081.

Medvedev, A.V., 2002. Epileptiform spikes desynchronize and diminish fast (gamma) activity of the brain: an anti-binding mechanism? Brain Res. Bull. 58 (1), 115-128.

Menon, V., Freeman, W.J., Cutillo, B.A., Desmond, J.E., Ward, M.F., Bressler, S.L., Laxer, K.D., Barbaro, N., Gevins, A.S., 1996. Spatio-temporal correlations in human gamma band electrocorticograms. Electroencephalogr. Clin. Neurophysiol. 98, 89-102.

Muller-Gass, A., Campbell, K., 2002. Event-related potential measures of the inhibition of information processing: I. Selective attention in the waking stage. Int. J. Psychophysiol. 46, 177-195.

Oswald, I., Taylor, A.M., Treisman, M., 1960. Discriminative response to stimulation during human sleep. Brain 83, 440-453.

Özdemir, A.K., Karakaș, S., Çakmak, E.D., Tüfekçi, D.İ., Arıkan, O., 2005. Time-frequency component analyzer and its application to brain oscillatory activity. J. Neurosci. Methods 145, 107-125.

Padmanabhapillai, A., Porjesz, B., Ranganathan, M., Jones, K.A., Chorlian, D. B., Tang, Y., Kamarajan, C., Rangaswamy, M., Stimus, A., Begleiter, H., 2005. Suppression of early evoked gamma band response in male alcoholics during a visual oddball task. Int. J. Psychophysiol.

Perrin, F., Garcia-Larrea, L., Mauguiere, F., Bastuji, H., 1999. A differential brain response to the subject's own name persists during sleep. Clin. Neurophysiol. 110, 2153-2164.

Sainsbury, R.S., 1998. Hippocampal theta: a sensory-inhibition theory of function. Neurosci. Biobehav. Rev. 22, 237-241.

Sakowitz, O.W., Quiroga, R.Q., Schurmann, M., Bașar, E., 2001. Bisensory stimulation increases gamma-responses over multiple cortical regions. Brain Res. Cogn. Brain Res. 11 (2), 267-279.

Schürmann, M., Bașar-Eroğlu, C., Demiralp, T., Bașar, E., 1995. A psychophysiological interpretation of theta and delta responses in cognitive event-related potential paradigms. In: Kruse, P., Stadler, M. (Eds.), Ambiguity in Mind and Nature. Springer Series in Synergetics, vol. 64. Springer-Verlag, Berlin.

Steraide, M., Amzica, F., 1996. Intracortical and corticothalamic coherency of fast spontaneous oscillations. Proc. Natl. Acad. Sci. U. S. A. 93, 2533-2538.

Tallon-Baudry, C., Bertrand, O., Delpuech, C., Pernier, J., 1996. Stimulusspecificity of phase-locked and nonphase-locked $40 \mathrm{~Hz}$ visual responses in humans. J Neurosci. 16, 4240-4249.

Vasey, M.W., Thayer, J.F., 1987. The continuing problem of false positives in repeated measures ANOVA in psychophysiology: a multivariate solution. Psychophysiology 24 (4), 479-486.

Weitzman, E.D., Kremen, H., 1965. Auditory evoked responses during different stages of sleep in man. Electroencephalogr. Clin. Neurophysiol. 18, 65-70.

Winter, O., Kok, A., Kenemans, J.L., Elton, M., 1995. Auditory event-related potentials to deviant stimuli during drowsiness and stage 2 sleep. Electroencephalogr. Clin. Neurophysiol. 96, 398-412. 